\title{
Dynamically generated open and hidden charm meson systems
}

\author{
D. Gamermann, ${ }^{1}$ E. Oset, ${ }^{1}$ D. Strottman, ${ }^{1}$ and M. J. Vicente Vacas ${ }^{1}$ \\ ${ }^{1}$ Departamento de Física Teórica and IFIC, Centro Mixto Universidad de Valencia-CSIC, Institutos de Investigación de Paterna, \\ Aptdo. 22085, 46071 Valencia, Spain
}

(Received 18 December 2006; revised manuscript received 25 June 2007; published 16 October 2007)

\begin{abstract}
We will study open and hidden charm scalar meson resonances within two different models. The first one is a direct application of a chiral Lagrangian already used to study flavor symmetry breaking in Skyrme models. In another approach to the problem a $S U(4)$ symmetric Lagrangian is built and the symmetry is broken down to $S U(3)$ by identifying currents where heavy mesons are exchanged and suppressing those. Unitarization in coupled channels leads to dynamical generation of resonances in both models, in particular, a new hidden charm resonance with mass $3.7 \mathrm{GeV}$ is predicted. The small differences between these models and with previous works is discussed. We also perform an error analysis of the results, checking their stability and determining the uncertainties in masses and couplings of the heavy resonances.
\end{abstract}

DOI: 10.1103/PhysRevD.76.074016

PACS numbers: $12.39 . \mathrm{Hg}$

\section{INTRODUCTION}

The discovery and soon after confirmation of charmed scalar resonances by BABAR and Belle [1-3] has opened a controversy about their structures. In the $q \bar{q}$ picture these resonances are naturally assigned as ${ }^{3} P_{0}$ states in the ${ }^{2 S+1} l_{j}$ notation, but calculations done long before in the framework of quark model potentials [4] had mass predictions which turned out to be more than $100 \mathrm{MeV}$ off the real mass of the states. Lattice calculations also fail in calculating the masses with a $q \bar{q}$ assignment [5].

This situation has sparked the discussion whether these resonances could have a different structure. Some authors have suggested a $q q \bar{q} \bar{q}$ structure [6,7] or a mixing between four quarks and the usual $q \bar{q}$ structure [8]. Also molecular states have been suggested [9-11]. For discussions on these and other controversial heavy mesons see Refs. [12-14].

Unitarized coupled channel models have also been considered for the study of these resonances in [15-17]. These works have used a chiral Lagrangian based on heavy quark symmetry [18-21] for the open charm sector which neglects exchanges of heavy vector mesons in the implicit Weinberg-Tomozawa term. We intend to extend the study for all possible sectors of the interaction, including the hidden charm and the double charmed sector. The exchange of heavy vector mesons is also taken into account in our approach, with the corresponding terms properly accounting for the larger mass of the heavy vector mesons.

In the present work we will construct a Lagrangian for the interaction of the 15-plet of pseudoscalar mesons in $S U(4)$. $S U(4)$ symmetry breaking will be considered by suppressing the exchange of heavy vector mesons. The $S U$ (3) structure of the interaction will be thoroughly analyzed and unitarization in coupled channels will lead to the generation of scalar resonances corresponding to poles in the T-matrix. For comparison, in the open charm sector, we will also solve the problem with yet another Lagrangian which has been considered in the study of flavor symmetry breaking effects in Skyrme models. This Lagrangian gives similar results, supporting our present model.

The work is organized as follows. In the next section a brief review of the structure of the $S U(4) 15$-plet will be presented. Section III is dedicated to the explanation of the construction of the Lagrangian and in Sec. IV the theoretical framework for solving the scattering equations in a unitarized approach is presented. Section V is dedicated to analyzing the results and a brief summary is presented in Sec. VI.

\section{THE 15-PLET}

In this work the framework already used to study the interaction of the octet of pseudoscalar mesons in $S U(3)$ [22] will be extended to include charmed mesons. This will involve some extrapolation to $S U(4)$. In the $q \bar{q}$ picture, mesons involving charm will be classified as $4 \otimes \overline{4}=15 \oplus$ 1 , hence belonging to a 15-plet or a singlet. It is interesting to understand how the 15 representation of $S U(4)$ breaks down into representations of $S U(3)$ and in which channels the interaction of the multiplets will be attractive or repulsive.

The pseudoscalar mesons are represented by a 15-plet of $S U(4)$ as shown in Fig. 1. Once $S U(4)$ symmetry is broken into $S U(3)$, the 15-plet breaks down into four multiplets of the lower symmetry, an octet, a triplet, an antitriplet and a singlet:

$$
15 \rightarrow 1 \oplus 3 \oplus \overline{3} \oplus 8 .
$$

The octet and the singlet have null charm quantum number, the triplet and the antitriplet have negative and positive charm quantum number, respectively. When studying the meson-meson interaction, one can decompose the scattering of two 15-plets of $S U(4)$ according to its $S U(3)$ inner structure, see Table I. 


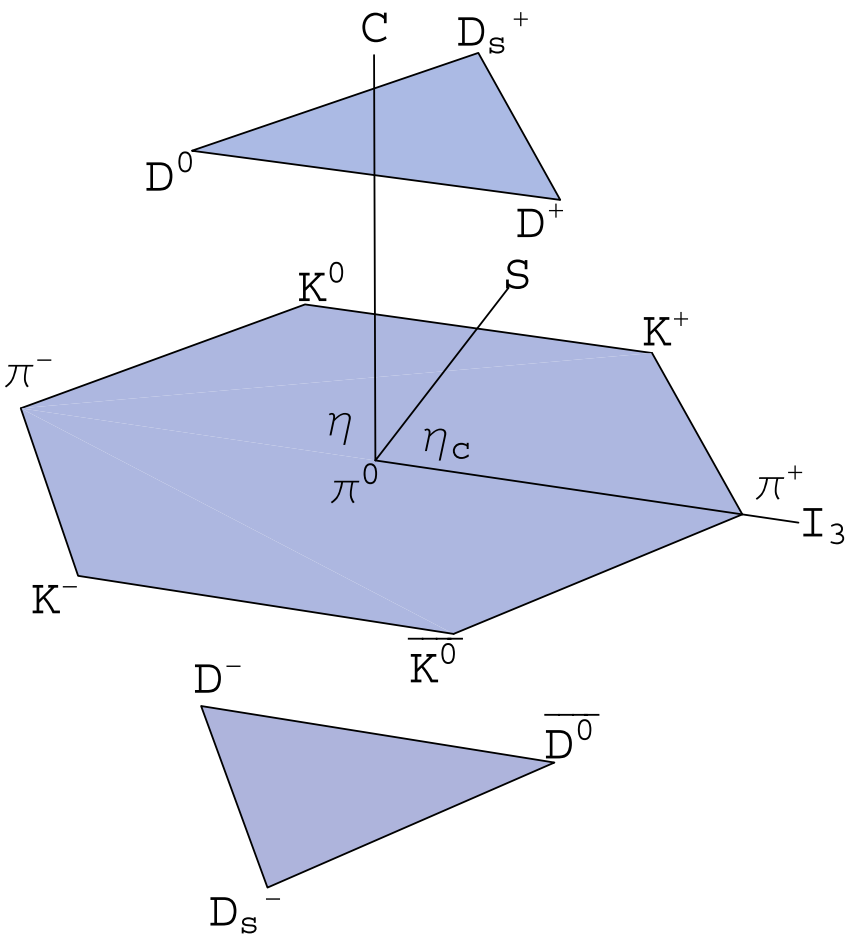

FIG. 1 (color online). 15-plet from $S U(4)$ with its mesons assignments.

The interaction $8 \otimes 8$ is already very well studied [2229]. It is already known that in $s$-wave it generates dynamically poles in the T-matrix which are associated with the $f_{0}(980), \kappa, a_{0}(980)$, and $\sigma$ resonances. Also the interaction $\overline{3} \otimes 8$ has already been studied [15-17] in some different approaches. In this sector, as we will show in the following sections, the interaction, when diagonalized in a $S U(3)$ basis, is attractive in $\overline{3}$ and 6 , while repulsive in the $\overline{15}$, so one could expect to generate five poles in $(S, I)$ : from the $\overline{3}$ with isospin 0 and $\frac{1}{2}$ and strangeness 1 and 0 , respectively; from the 6 with $I=0, \frac{1}{2}, 1$ and $S=-1,0,1$, respectively. Moreover, the interaction in the $\overline{3} \otimes 3$ is attractive in both the 8 and the 1 , so one can, in principle, expect four new resonances in the hidden charm sector.

TABLE I. $S U(3)$ decomposition of the meson-meson interaction in $S U(4)$. The sectors not shown in the table correspond to the $C=-1,-2$ states which are just charge conjugate states (antiparticles) from the ones shown.

\begin{tabular}{cc}
\hline \hline Charm & Interacting multiplets \\
\hline 2 & $\overline{3} \otimes \overline{3} \rightarrow 3 \oplus \overline{6}$ \\
1 & $\overline{3} \otimes 8 \rightarrow \overline{1} 5 \oplus \overline{3} \oplus 6$ \\
$\overline{3} \otimes 1 \rightarrow \overline{3}$ \\
$\overline{3} \otimes 3 \rightarrow 8 \oplus 1$ \\
$1 \otimes 1 \rightarrow 1$ \\
& $8 \otimes 1 \rightarrow 8$ \\
& $8 \otimes 8 \rightarrow 1 \oplus 8_{s} \oplus 8_{a} \oplus 10 \oplus \overline{10} \oplus 27$ \\
\hline \hline
\end{tabular}

The interaction in the $C=2$ sector is repulsive in the $\overline{6}$ and in the 3 the interaction vanishes.

Apart from studying the different sectors separately, it is interesting to see how the mixing of states from different sectors with the same $S U(3)$ representation of Table I affects the interaction.

Furthermore, if heavy resonances are generated from the $3 \otimes \overline{3}$ one can expect, in principle, that the mixing of those heavy channels with light ones coming from $8 \otimes 8$ will make its width quite large because of the large phase-space available for decay. However, we shall also see that there are subtleties in the interaction which suppress these decays.

\section{THE LAGRANGIANS}

The $S U(3)$ lowest order chiral Lagrangian reads [30,31]:

$$
\mathcal{L}_{\chi}=\frac{f_{\pi}^{2}}{4} \operatorname{Tr}\left(\partial_{\mu} U \partial^{\mu} U\right)+\frac{f_{\pi}^{2} m_{\pi}^{2}}{4} \operatorname{Tr}\left(U+U^{\dagger}-2\right),
$$

where $U$ is the field containing the pseudoscalar mesons from the $S U(3)$ octet and $\mathrm{Tr}$ represents a trace in flavor space:

$$
\begin{gathered}
U=e^{i \sqrt{2} \phi_{8} / f_{\pi},} \\
\phi_{8}=\left(\begin{array}{ccc}
\frac{\pi^{0}}{\sqrt{2}}+\frac{\eta}{\sqrt{6}} & \pi^{+} & K^{+} \\
\pi^{-} & \frac{-\pi^{0}}{\sqrt{2}}+\frac{\eta}{\sqrt{6}} & K^{0} \\
K^{-} & \bar{K}^{0} & \frac{-2 \eta}{\sqrt{6}}
\end{array}\right) .
\end{gathered}
$$

Flavor symmetry breaking effects can be introduced with two new terms in the Lagrangian [32,33]:

$$
\begin{gathered}
\mathcal{L}_{\mathrm{SB}}=\frac{f_{K}^{2} m_{K}^{2}-f_{\pi}^{2} m_{\pi}^{2}}{6} \operatorname{Tr}\left(\left(\hat{1}-\sqrt{3} \lambda_{8}\right)\left(U+U^{\dagger}-2\right)\right) \\
-\frac{f_{K}^{2}-f_{\pi}^{2}}{12} \operatorname{Tr}\left(\left(\hat{1}-\sqrt{3} \lambda_{8}\right)\left(U l_{\mu} l^{\mu}+l_{\mu} l^{\mu} U^{\dagger}\right)\right) \\
l_{\mu}=U^{\dagger} \partial_{\mu} U
\end{gathered}
$$

where $\lambda_{8}$ is one of the $S U(3)$ generators.

In [34] these Lagrangians are extended to $S U(n)$. In this new approach the symmetry breaking sector is written as

$$
\begin{aligned}
\mathcal{L}_{\mathrm{SB}}= & \frac{1}{8} \sum_{k=3}^{n} \gamma_{k} \operatorname{Tr}\left(\left(\hat{1}-\sqrt{\frac{1}{2} k(k-1)} \lambda_{k^{2}-1}\right)\right. \\
& \left.\times\left(U l_{\mu} l^{\mu}+l_{\mu} l^{\mu} U^{\dagger}\right)\right) \\
& +\frac{1}{8} \sum_{k=3}^{n} \delta_{k} \operatorname{Tr}\left(\left(\hat{1}-\sqrt{\frac{1}{2} k(k-1)} \lambda_{k^{2}-1}\right)\right. \\
& \left.\times\left(U+U^{\dagger}-2\right)\right),
\end{aligned}
$$

but now $U$ belongs to a $S U(n)$ representation. 
By expanding the $U$ matrix until fourth order in the meson fields, one can identify the mass and kinetic terms for each field and fix the symmetry breaking parameters for $S U(4)$ and $S U(3)$ as

$$
\begin{gathered}
\gamma_{3}=\frac{4}{6}\left(f_{K}^{2}-f_{\pi}^{2}\right), \\
\delta_{3}=\frac{4}{3}\left(f_{K}^{2} m_{K}^{2}-f_{\pi}^{2} m_{\pi}^{2}\right), \\
\gamma_{4}=\frac{1}{2}\left(f_{D}^{2}+f_{K}^{2}-2 f_{\pi}^{2}\right), \\
\delta_{4}=f_{D}^{2} m_{D}^{2}-\frac{1}{3} f_{K}^{2} m_{K}^{2}-\frac{1}{3} f_{\pi}^{2} m_{\pi}^{2} .
\end{gathered}
$$

In this work we will consider only the difference between $f_{D}$ and $f_{\pi}$ which is about $70 \%$ and we will make the approximation $f_{K}=f_{\pi}$.

For constructing our model we will first consider a $S U(4)$ field containing all fields from the 15 -plet ${ }^{1}$ :

$$
\begin{aligned}
\Phi & =\sum_{i=1}^{15} \frac{\varphi_{i}}{\sqrt{2}} \lambda_{i} \\
= & \left(\begin{array}{cccc}
\frac{\pi^{0}}{\sqrt{2}}+\frac{\eta}{\sqrt{6}}+\frac{\eta_{c}}{\sqrt{12}} & \pi^{+} & K^{+} & \bar{D}^{0} \\
\pi^{-} & \frac{-\pi^{0}}{\sqrt{2}}+\frac{\eta}{\sqrt{6}}+\frac{\eta_{c}}{\sqrt{12}} & K^{0} & D^{-} \\
K^{-} & \bar{K}^{0} & \frac{-2 \eta}{\sqrt{6}}+\frac{\eta_{c}}{\sqrt{12}} & D_{s}^{-} \\
D^{0} & D^{+} & D_{s}^{+} & \frac{-3 \eta_{c}}{\sqrt{12}}
\end{array}\right) .
\end{aligned}
$$

A current is then defined:

$$
J_{\mu}=\left[\partial_{\mu} \Phi, \Phi\right],
$$

and a Lagrangian is built by connecting two currents and adding an extra term proportional to the square mass of the fields:

$$
\mathcal{L}_{P P P P}=\frac{1}{12 f^{2}} \operatorname{Tr}\left(J_{\mu} J^{\mu}+\Phi^{4} M\right) .
$$

$S U(4)$ and $S U(3)$ flavor symmetry breaking already arise from the mass term when the matrix $M$ is not proportional to the identity matrix. We take

$$
M=\left(\begin{array}{cccc}
m_{\pi}^{2} & 0 & 0 & 0 \\
0 & m_{\pi}^{2} & 0 & 0 \\
0 & 0 & 2 m_{K}^{2}-m_{\pi}^{2} & 0 \\
0 & 0 & 0 & 2 m_{D}^{2}-m_{\pi}^{2}
\end{array}\right)
$$

The term with the matrix $M$ is exactly the same one appearing in the chiral Lagrangian of Eq. (2) after breaking $S U(4)$ and $S U(3)$ by means of (7). The term $J_{\mu} J^{\mu}$ in Eq. (14) appears for four meson fields from the kinetic

\footnotetext{
${ }^{1}$ What here is called $\eta$ and $\eta_{c}$ are actually $\eta_{8}$ and $\eta_{15}$ states, which mix with a singlet, $\eta_{1}$, to form the physical states $\eta, \eta^{\prime}$, and $\eta_{c}$, but in this work this mixing will not be taken into account, and it will be considered that the physical states are just described by their most important components.
}

term, $\partial_{\mu} U \partial^{\mu} U$, of the chiral Lagrangian in Eq. (2) if $U$ is taken as a $S U(4)$ representation by means of replacing $\phi_{8}$ in Eq. (4) by $\Phi$ in Eq. (12).

We will also implement other different sources of $S U(4)$ flavor symmetry breaking in a way that we explain below.

The constant $f$ appearing in the Lagrangian (14) is, in principle, the pion decay constant (in this work $f_{\pi}=$ 93.0 MeV). However, a different one will be used for the heavy mesons. In this latter case, the $f^{2}$ appearing in the amplitudes should be thought of as the product of $\sqrt{f}$ for each meson leg in the corresponding vertex, with $f=$ $f_{\pi}=93.0 \mathrm{MeV}$ for light mesons and $f=f_{D}=165 \mathrm{MeV}$ for heavy ones. This value for $f_{D}$ is of the order of magnitude expected from the experimental point of view [35] and lattice calculations [36]. Yet, in Sec. V B, we will study the theoretical uncertainties and the stability of the generated heavy resonances by varying these parameters among others still to be introduced.

Directly applying Feynman rules to obtain transition amplitudes from this Lagrangian would be too much of a simplification. Indeed, the term $J_{\mu} J^{\mu}$ of the chiral Lagrangian is usually visualized as the exchange of a vector meson between pairs of pseudoscalar fields in the limit of $q^{2} \ll m_{V}^{2}$ (the Weinberg-Tomozawa term). In this case the kinetic term of the Lagrangian of Eq. (14) is $S U(4)$ flavor symmetric and therefore implicitly assumes equal $m_{V}$ for all the exchanges of heavy and light vector mesons. In Refs. [15,17] an $S U(3)$ version of the interaction based on heavy quark symmetry is used which would correspond to allowing the exchange of only light vector mesons in the Weinberg-Tomozawa Lagrangian described by the derivative term of Eq. (14), and neglecting the $M$ term [37]. In the present work we shall go one step further by allowing also the exchange of heavy vector mesons but weighted by their respective squared masses and we shall also keep the mass term as done in [32-34]. In order to implement this we first decompose the $\Phi$ field into its $S U(3)$ components:

$$
\Phi=\left(\begin{array}{cc}
\phi_{8}+\frac{1}{\sqrt{12}} \phi_{1} \hat{1}_{3} & \phi_{3} \\
\phi_{\overline{3}} & -\frac{3}{\sqrt{12}} \phi_{1}
\end{array}\right) .
$$

The $\hat{1}_{3}$ is the $3 \times 3$ identity matrix and the fields $\phi_{i}$ contain the meson fields for each $i$-plet of $S U$ (3) into which the 15-plet of $S U(4)$ decomposes:

$$
\begin{gathered}
\phi_{8}=\left(\begin{array}{ccc}
\frac{\pi^{0}}{\sqrt{2}}+\frac{\eta}{\sqrt{6}} & \pi^{+} & K^{+} \\
\pi^{-} & \frac{-\pi^{0}}{\sqrt{2}}+\frac{\eta}{\sqrt{6}} & K^{0} \\
K^{-} & \bar{K}^{0} & \frac{-2 \eta}{\sqrt{6}}
\end{array}\right), \\
\phi_{3}=\left(\begin{array}{c}
\bar{D}^{0} \\
D^{-} \\
D_{s}^{-}
\end{array}\right), \\
\phi_{\overline{3}}=\left(\begin{array}{lll}
D^{0} & D^{+} & D_{s}^{+}
\end{array}\right),
\end{gathered}
$$




$$
\phi_{1}=\eta_{c} .
$$

In this way the Lagrangian in (14) can be decomposed into six parts:

$$
\begin{gathered}
\mathcal{L}_{P P P P}=\frac{1}{12 f^{2}}\left(\mathcal{L}_{8}+\mathcal{L}_{3}+\mathcal{L}_{31}+\mathcal{L}_{83}+\mathcal{L}_{831}+\mathcal{L}_{\text {mass }}\right), \\
\mathcal{L}_{8}=\operatorname{Tr}\left(J_{88 \mu} J_{88}^{\mu}\right), \\
\mathcal{L}_{3}=J_{\overline{3} 3 \mu} J_{\overline{3} 3}^{\mu}+\operatorname{Tr}\left(J_{3 \overline{3} \mu} J_{3 \overline{3}}^{\mu}\right), \\
\mathcal{L}_{31}=\frac{8}{3} J_{\overline{3} 1 \mu} J_{13}^{\mu}, \\
\mathcal{L}_{83}=2\left(J_{\overline{3} 8 \mu} J_{83}^{\mu}+\operatorname{Tr}\left(J_{3 \overline{3} \mu} J_{88}^{\mu}\right)\right), \\
\mathcal{L}_{831}=\frac{4}{\sqrt{3}}\left(J_{\overline{3} 1 \mu} J_{83}^{\mu}+J_{\overline{3} 8 \mu} J_{13}^{\mu}\right), \\
\mathcal{L}_{\text {mass }}=\operatorname{Tr}\left(M \Phi^{4}\right),
\end{gathered}
$$

where the currents are defined as $J_{i j}^{\mu}=\left(\partial^{\mu} \phi_{i}\right) \phi_{j}-$ $\phi_{i}\left(\partial^{\mu} \phi_{j}\right)$.

Now the exchange of charmed (heavy) vector mesons can be easily identified in the different pieces of the Lagrangian by identifying currents carrying explicitly charm quantum number. The $\mathcal{L}_{8}$ term accounts for the exchange of light vector mesons only. In $\mathcal{L}_{83}$ the first term is mediated by heavy vector mesons and the second term by light ones, $\mathcal{L}_{831}$ and $\mathcal{L}_{31}$ have only contributions from heavy vector mesons, and $\mathcal{L}_{3}$ will still have to be worked out further.

The separation of the heavy vector-meson contribution from $\mathcal{L}_{3}$ is more subtle because the exchange of a heavy hidden charm meson in this sector occurs in charge and flavor conserving hadronic currents, where the $\rho_{0}$ and $\omega$ also contribute. The strategy followed here is to construct a Lagrangian connecting the current $J_{\mu}$ with a vector field $V^{\mu}[37,38]:$

$$
\mathcal{L}_{P P V}=-\frac{i g}{\sqrt{2}} \operatorname{Tr}\left(\left[\partial_{\mu} \Phi, \Phi\right] V^{\mu}\right) .
$$

Here $V^{\mu}$ is a $4 \times 4$ matrix with the same structure as $\Phi$, but with the 15-plet of vector mesons instead. The heavy vector meson which can be exchanged in charge and flavor conserving hadronic currents is the $J / \psi$.

The $J / \psi$ contribution can be calculated from the Lagrangian (24) and it is easy to see that when the vector mesons are connecting equal hadronic currents one has a contribution with weights $\frac{1}{3}$ and $\frac{2}{3}$ for light vector mesons and the $J / \psi$, respectively, while the weights are $-\frac{1}{3}$ and $\frac{4}{3}$ in terms connecting different currents. Appendix $\mathrm{C}$ shows in more detail how to work out $\mathcal{L}_{3}$.
With all these considerations the full Lagrangian can now be rewritten in terms of the correction parameters:

$$
\begin{gathered}
\gamma=\left(\frac{m_{L}}{m_{H}}\right)^{2}, \\
\psi_{3}=\frac{1}{3}+\frac{2}{3}\left(\frac{m_{L}}{m_{J / \psi}}\right)^{2}, \\
\psi_{5}=-\frac{1}{3}+\frac{4}{3}\left(\frac{m_{L}}{m_{J / \psi}}\right)^{2} .
\end{gathered}
$$

Here $m_{L}$ and $m_{H}$ are parameters to represent the masses of light and heavy vector mesons, respectively. In a first approximation, they will be set to $m_{L}=800 \mathrm{MeV}$ and $m_{H}=2050 \mathrm{MeV}$. With these ingredients the full corrected Lagrangian can be written as

$$
\begin{aligned}
\mathcal{L}= & \frac{1}{12 f^{2}}\left(\operatorname{Tr}\left(J_{88 \mu} J_{88}^{\mu}+2 J_{3 \overline{3} \mu} J_{88}^{\mu}+J_{3 \overline{3}_{\mu}} J_{3 \overline{3}}^{\mu}\right)\right. \\
& +\frac{8}{3} \gamma J_{\overline{3} 1 \mu} J_{13}^{\mu}+\frac{4}{\sqrt{3}} \gamma\left(J_{\overline{3} 1 \mu} J_{83}^{\mu}+J_{\overline{3} 8 \mu} J_{13}^{\mu}\right) \\
& \left.+2 \gamma J_{\overline{3} 8 \mu} J_{83}^{\mu}+\psi_{5} J_{\overline{3} 3 \mu} J_{\overline{3} 3}^{\mu}+\mathcal{L}_{\text {mass }}\right) .
\end{aligned}
$$

Note that from Eq. (28) we can recover the usual lowest order chiral Lagrangian for $S U(3)$, which is the term proportional to $\operatorname{Tr}\left(J_{88 \mu} J_{88}^{\mu}\right)$, while the Lagrangian used by Kolomeitsev [15] and Guo [17], based on heavy quark symmetry [18-21], is proportional to the term $\operatorname{Tr}\left(J_{3 \overline{3} \mu} J_{88}^{\mu}\right)$. Our model has also terms for the interaction of heavy mesons only, proportional to $\operatorname{Tr}\left(J_{3 \overline{3} \mu} J_{3 \overline{3}}^{\mu}\right)$ and $J_{\overline{3} 3 \mu} J_{\overline{3} 3}^{\mu}$ and all the other terms are corrections that can be controlled by the parameter $\gamma$.

From this Lagrangian, applying the usual Feynman rules, the transition amplitudes in Appendix A are calculated and used as potentials for each possible reaction. These potentials, projected in $s$-wave, will be used as the kernel for solving the scattering equation.

In order to support our results, also the chiral Lagrangian with the flavor symmetry breaking pieces will be used to solve the scattering problem in the open charm sector. Very similar results are found and will be discussed in Sec. V.

\section{THE SCATTERING PROBLEM}

The amplitudes needed, $\mathcal{M}(s, \theta)$, are written in Appendix A for the Lagrangian of Eq. (28). Since we are only interested in $s$-wave meson-meson scattering, we first project the amplitudes over $s$-waves, by making a simple angular integration. After projecting the amplitudes for $s$-wave they will be transformed to isospin basis and inserted into the Bethe-Salpeter equation which in the on-shell formalism of [22,39] is reduced to an algebraic equation:

$$
T=V+V G T .
$$


In this equation $V$ is the potential, a matrix constructed with the tree level transition amplitudes for each one of the possible channels, projected over $s$-wave. The matrix $G$ is diagonal with each one of its nonzero elements given by the loop function for the two particles in each channel:

$$
\begin{aligned}
& G_{i i}=i \int \frac{d q^{4}}{(2 \pi)^{4}} \frac{1}{q^{2}-m_{1}^{2}+i \epsilon} \frac{1}{(P-q)^{2}-m_{2}^{2}+i \epsilon}= \\
& \frac{1}{16 \pi^{2}}\left(\alpha_{i}+\log \frac{m_{1}^{2}}{\mu^{2}}+\frac{m_{2}^{2}-m_{1}^{2}+s}{2 s} \log \frac{m_{2}^{2}}{m_{1}^{2}}+\frac{p}{\sqrt{s}}\right. \\
& \quad \times\left(\log \frac{s-m_{2}^{2}+m_{1}^{2}+2 p \sqrt{s}}{-s+m_{2}^{2}-m_{1}^{2}+2 p \sqrt{s}}\right. \\
& \left.\left.\quad+\log \frac{s+m_{2}^{2}-m_{1}^{2}+2 p \sqrt{s}}{-s-m_{2}^{2}+m_{1}^{2}+2 p \sqrt{s}}\right)\right) .
\end{aligned}
$$

$P$ in Eq. (30) is the total four-momentum of the two mesons in channel $i$ and $m_{1}$ and $m_{2}$ are the masses of the two mesons in this channel. The expression in Eq. (31) is calculated using dimensional regularization. Over the real axis $p$ is the three-momentum of the mesons in the center of mass frame:

$$
p=\frac{\sqrt{\left(s-\left(m_{1}+m_{2}\right)^{2}\right)\left(s-\left(m_{1}-m_{2}\right)^{2}\right)}}{2 \sqrt{s}} .
$$

In the complex plane the momentum $p$ is calculated using the same expression. Equation (29) with Eqs. (30) and (31) makes implicit use of dispersion relations in which only the right-hand (physical) cut is considered. It was proved in [40] that the left-hand cut provides a mod-
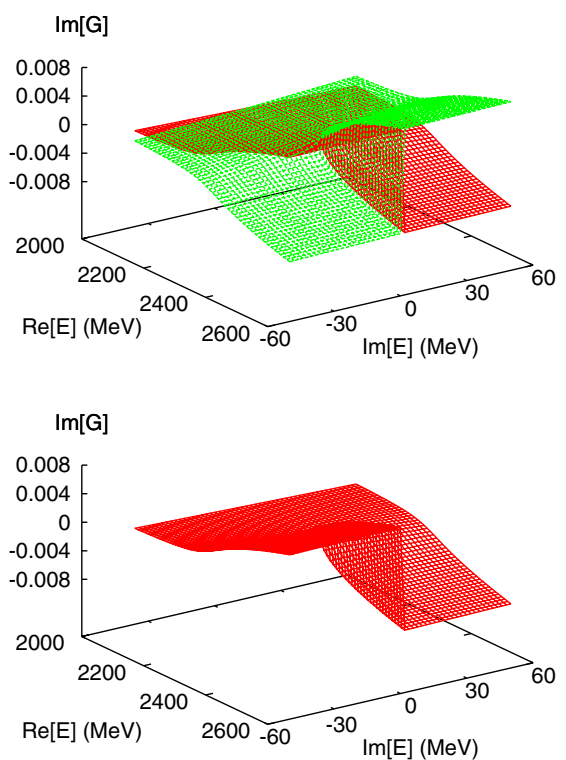

erate contribution, and more important, very weakly energy dependent, such that its contribution can be easily accommodated in terms of the subtraction constant that we use, in the range of energies of interest to us.

In this work we will set the loop parameter in Eq. (31) to $\mu=1500 \mathrm{MeV}$ and fit the subtraction constant, $\alpha$, as a free parameter.

This loop function has the right imaginary part to ensure the unitarity of the T-matrix [23]:

$$
\operatorname{Im}\left(G_{i i}\right)=-\frac{p}{8 \pi \sqrt{s}} .
$$

Equation (29) can be easily inverted:

$$
T=(\hat{1}-V G)^{-1} V .
$$

When looking for poles in the complex plane one should be careful because of the cuts of the loop function beyond each threshold. Bound states appear as poles over the real axis and below threshold in the first Riemann sheet. Resonances show themselves as poles above threshold and in the second Riemann sheet of the channels which are open.

Over the real axis the discontinuity of the loop function is known to be 2 times its imaginary part [41] so, knowing the value of the imaginary part of the loop function over the axis, Eq. (33), one can do a proper analytic continuation of it for the whole complex plane:

$$
G_{i i}^{I I}=G_{i i}^{I}+i \frac{p}{4 \pi \sqrt{s}}, \quad \operatorname{Im}(p)>0 .
$$

$G^{I I}$ and $G^{I}$ refer to the loop function in the second and first Riemanian sheets, respectively.
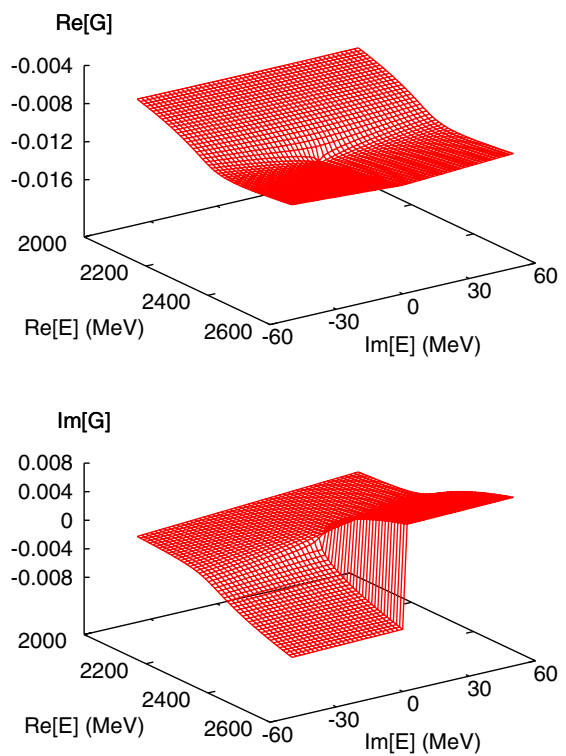

FIG. 2 (color online). Upper left: Imaginary part of the loop on the first and second Riemann sheets superposed. Upper right: Real part of the loop in the first Riemann sheet. Bottom left, right are the imaginary part of the loop in the first and second Riemann sheets, respectively. 
Figure 2 shows some plots of the loop function in the complex plane.

\section{RESULTS}

The amplitudes listed in Appendix A are in a charge basis. First, they are transformed to an isospin basis and then to a $S U(3)$ basis by means of the isospin and $S U(3)$ states given in Appendix B. The $S U(3)$ symmetry breaking is then studied. The amplitudes in a $S U(3)$ basis show in which sectors the interaction is attractive (where it may generate resonances). The most important term in each amplitude is the $s$ term, so when an amplitude has a negative factor multiplying $s$ it is considered to be attractive. The results of the diagonalization of the interaction in $S U(3)$ basis are as follows:

$8 \otimes 8 \rightarrow 1 \oplus 8_{s} \oplus 8_{a} \oplus 10 \oplus \overline{10} \oplus 27$ : The interaction here is repulsive in the 27; there is no interaction in the 10 nor in the $\overline{10}$; in one of the octets, because of its symmetry properties [here we should consider scattering of identical particles if $S U(3)$ symmetry is restored] there is no interaction in even $l$ partial waves; in the other octet and in the singlet there is attraction, which will lead to the formation of 4 states, to be identified as the light scalar resonances, $\sigma, f_{0}, a_{0}$, and $\kappa$.

$\overline{3} \otimes \overline{3} \rightarrow 3 \oplus \overline{6}$ : Here there is no interaction in the 3 when the correction parameters are set to 1 , otherwise the interaction has a $p$-wave structure $(t-u)$. In the sextet the interaction is repulsive, therefore, no double charmed scalar resonances are expected from our model.

$\overline{3} \otimes 3 \rightarrow 8 \oplus 1$ : The interaction is attractive in both the octet and the singlet if the correction parameters $\left(\psi_{3}, \psi_{5}\right)$ are set to 1 . In this case, where the large mass of the $J / \psi$ is disregarded, one can see resonances generated. However, since the terms with the heavy vector meson have the largest weight in the amplitude, when the correction parameters are considered to take into account the different masses of the exchanged vector mesons, the resonances disappear for the octet. The singlet is always attractive irrespective to the correction parameters.

$\overline{3} \otimes 8 \rightarrow \overline{3} \oplus 6 \oplus \overline{15}$ : In the antitriplet and sextet there is attraction while in the $\overline{15}$-plet there is repulsion. We generate in our model five resonances with charmed quantum number, two from the antitriplet and three from the sextet.

We discuss below the free parameters of the theoretical framework and how we fit them. The parameters fitted are $\alpha_{H}$ and $\alpha_{L}$. The $\alpha$ 's are the subtraction constants for the loop functions. The parameter $\alpha_{H}$ was chosen for channels involving at least one heavy pseudoscalar meson and a different one for channels where there are just light ones, $\alpha_{L}$.

One of the novel aspects of the present work is that we allow the mixing of the light mesons with the heavy ones in the search of zero charm or hidden charm scalar mesons. The first interesting result is that the influence of the heavy meson sector in the generation of the light scalar reso- nances $\left(\sigma, f_{0}, a_{0}, \kappa\right)$ is negligible. For instance it was checked that different values $\alpha_{H}$ have very small effect over the pole position for the light resonances. Varying $\alpha_{H}$ between -0.3 and -2.3 has less than $10 \%$ effect over the pole position of the $f_{0}$ resonance, for example. So the heavy sector can be worked independently from the light one. Although the main aspect of this work is the study of the heavy resonances, we also present results for the light sector for completeness, since in the $\mathrm{C}=0, \mathrm{~S}=0, \mathrm{I}=0$ sector light channels are indeed included in the coupled channel space in the generation of a hidden charm resonance.

With this in mind the open charm $(\mathrm{C}=1)$ sector was used to fit $\alpha_{H}$ so that the position of the pole in the $\mathrm{S}=1$, $\mathrm{I}=0$ sector matches the $D_{s 0}^{*}(2317)$, which has already been suggested as being dynamically generated in [1517]. After fixing the heavy parameter, the $\alpha_{L}$ was fitted by locating the pole position in the sector $\mathrm{C}=0, \mathrm{~S}=0, \mathrm{I}=$ 1 , which correspond to the $a_{0}$ resonance. We also made the fit of $\alpha_{H}$ for the model involving the chiral Lagrangian. The results are as follows:

Phenomenological model: $\alpha_{H}=-1.3$ and $\alpha_{L}=-1.3$.

Chiral model: $\alpha_{H}=-1.15$ (we only applied this model for the open charm sector).

This value of $\alpha_{H}$ for the phenomenological model is indicative and, in Sec. V B, we will study the effects in the heavy resonances of its variation.

\section{A. $S U(3)$ symmetry breaking}

In our phenomenological model it is assumed that the $S U$ (3) flavor symmetry breaking arises from the different masses of the interacting mesons. The mass used for each member of the 15-plet is $m_{\pi}=138.0 \mathrm{MeV}, m_{K}=$ $495.0 \mathrm{MeV}, \quad m_{\eta}=548.0 \mathrm{MeV}, \quad m_{D}=1865.0 \mathrm{MeV}$, $m_{D_{s}}=1968.0 \mathrm{MeV}$, and $m_{\eta_{c}}=2979.0 \mathrm{MeV}$.

Note that there is no isospin breaking in the model, all particles in a same isospin multiplet are considered to have the same mass. So, in this work, the Bethe-Salpeter equation, Eq. (29), was solved with $V$ in isospin basis.

$S U(3)$ symmetry can then be gradually broken by means of a symmetry breaking parameter $x$ which takes values between 1 and 0,1 meaning symmetry broken as we see it in the real world, and 0 symmetry restored. The masses of the mesons as a function of the parameter $x$ are given by

$$
m(x)=\bar{m}+x\left(m_{\text {phys. }}-\bar{m}\right),
$$

where $\bar{m}$ is the meson mass in the symmetry limit.

Two different values of $\bar{m}$ were used: for the light mesons (the ones belonging to the octet) it was set to $430 \mathrm{MeV}$ and for the heavy ones, $1900 \mathrm{MeV}$.

Also the correction parameters were changed along with $x$, although they just violate $S U(4)$ symmetry:

$$
\gamma(x)=1+x\left(\gamma_{\text {phys }}-1\right) .
$$


Similar functions are constructed for $\psi_{3}$ and $\psi_{5}$.

All scalar resonances in the same multiplet have the same mass once $S U(3)$ is restored while its breaking splits the masses of the different isospin multiplets. So, when written in the $S U(3)$ basis, the nondiagonal elements of the matrix $V$ [the ones which represent mixing between different $S U(3)$ multiplets] are always proportional to $m_{\pi}^{2}-m_{K}^{2}$.

Figures 3 and 4 show the pole positions in the $\mathrm{C}=0$ and $\mathrm{C}=1$ sectors, varying $x$ from 0 to 1 in steps of 0.2 . We should note that some resonances, for example, the $\kappa$ and the $D_{0}^{*}(2400)$ appear as cusps for small values of $x$. This happens because thresholds appear during the symmetry breaking procedure.

Table II displays the experimental situation of the scalar resonances, Table III shows the results in the open charm sector for the problem solved with the chiral Lagrangian

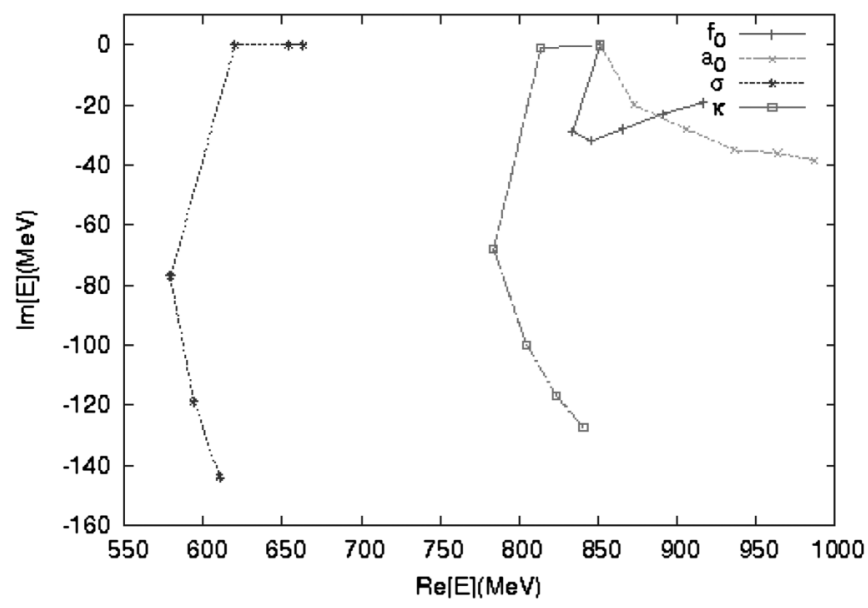

FIG. 3. Light scalars. The octet starts from $851.76 \mathrm{MeV}$ and the singlet from $663.13 \mathrm{MeV}$.

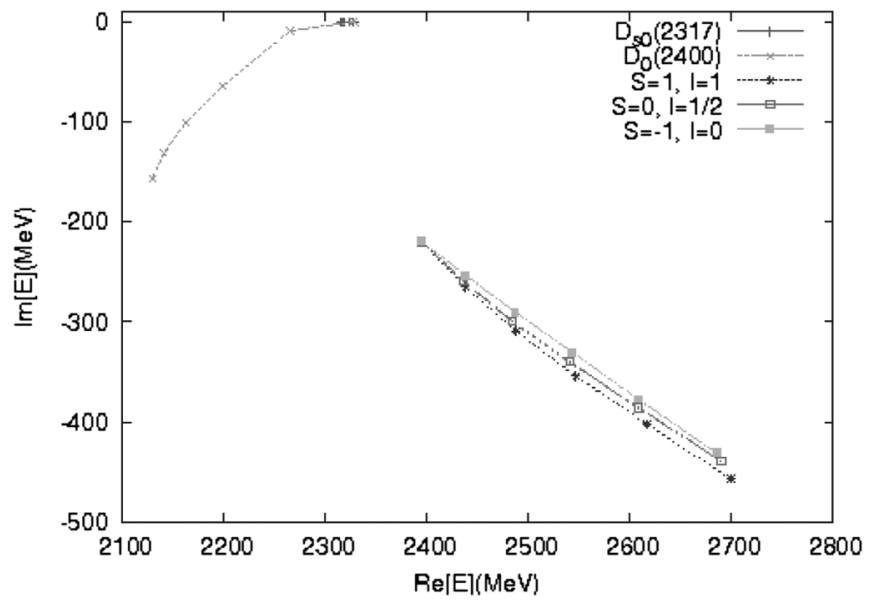

FIG. 4. Heavy scalars. The antitriplet starts from $2327.96 \mathrm{MeV}$ and the sextet from $(2394.87-i 219.33) \mathrm{MeV}$.
TABLE II. Data from [35].

\begin{tabular}{|c|c|c|c|c|}
\hline Resonance ID & $\mathrm{C} \mathrm{S}$ & I & Mass (MeV) & $\Gamma(\mathrm{MeV})$ \\
\hline$f_{0}$ & 00 & 0 & $980 \pm 10$ & $40-100$ \\
\hline$\sigma$ & 00 & 0 & $400-1200$ & $250-500$ \\
\hline$a_{0}$ & 00 & 1 & $984.7 \pm 1.2$ & $50-100$ \\
\hline$\kappa$ & $\begin{array}{ll}0 & 1\end{array}$ & $\frac{1}{2}$ & $841 \pm 30_{-73}^{+81}$ & $618 \pm 90_{-144}^{+96}$ \\
\hline$D_{s 0}^{*}(2317)$ & 11 & 0 & $2317.3 \pm 0.4 \pm 0.8$ & $<4.6$ \\
\hline$D_{0}^{*}(2400)$ & 10 & $\frac{1}{2}$ & $\begin{array}{c}2403 \pm 14 \pm 35 \\
2352 \pm 50\end{array}$ & $\begin{array}{c}283 \pm 24 \pm 34 \\
261 \pm 50\end{array}$ \\
\hline
\end{tabular}

TABLE III. Pole positions for the chiral Lagrangian.

\begin{tabular}{lccc|c|c}
\hline \hline Resonance ID & $\mathrm{C}$ & $\mathrm{S}$ & $\mathrm{I}$ & $\mathrm{RE}(\sqrt{s})(\mathrm{MeV})$ & $\mathrm{IM}(\sqrt{s})(\mathrm{MeV})$ \\
\hline$D_{s 0}^{*}(2317)$ & 1 & 1 & 0 & 2315.41 & 0 \\
$D_{0}^{*}(2400)$ & 1 & 0 & $\frac{1}{2}$ & 2147.65 & -107.29 \\
$(?)$ & 1 & 0 & $\frac{1}{2}$ & Cusp & Broad \\
$(?)$ & 1 & 1 & 1 & 2427.70 & -248.40 \\
$(?)$ & 1 & -1 & 0 & 2410.26 & -193.80 \\
\hline \hline
\end{tabular}

TABLE IV. Pole positions for the phenomenological model.

\begin{tabular}{lccc|c|c}
\hline \hline Resonance ID & $\mathrm{C}$ & $\mathrm{S}$ & $\mathrm{I}$ & $\mathrm{RE}(\sqrt{s})(\mathrm{MeV})$ & $\mathrm{IM}(\sqrt{s})(\mathrm{MeV})$ \\
\hline$f_{0}$ & 0 & 0 & 0 & 918.45 & -18.76 \\
$\sigma$ & 0 & 0 & 0 & 616.19 & -143.77 \\
$(?)$ & 0 & 0 & 0 & 3718.93 & -0.06 \\
$a_{0}$ & 0 & 0 & 1 & 987.68 & -38.29 \\
$\kappa$ & 0 & 1 & $\frac{1}{2}$ & 831.58 & -147.24 \\
$D_{s 0}^{*}(2317)$ & 1 & 1 & 0 & 2317.25 & 0 \\
$D_{0}^{*}(2400)$ & 1 & 0 & $\frac{1}{2}$ & 2129.26 & -157.00 \\
$(?)$ & 1 & 0 & $\frac{1}{2}$ & 2694.69 & -441.89 \\
$(?)$ & 1 & 1 & 1 & 2704.31 & -459.50 \\
$(?)$ & 1 & -1 & 0 & 2709.39 & -445.73 \\
\hline \hline
\end{tabular}

and Table IV shows the pole positions found within the phenomenological model developed in this work.

These results were obtained using for the parameters of the theory the ones presented until now along the paper. In the next subsection we will study, for our phenomenological model, the theoretical uncertainties produced in the heavy sector by changing these parameters.

\section{B. Theoretical uncertainties}

The true free parameters in our model are the $\alpha$ subtraction constants in the loop functions, all other parameters are meson masses or meson decay constants which are, in principle, fixed by experiment. In chiral models, chiral symmetry is tied to the use of the function $U=e^{i \sqrt{2} \phi / f}$, which requires the use of just one $f$, usually $f_{\pi}$, in the different amplitudes. Of course this symmetry is partially broken and in practice one has different values of $f$ for different mesons. For instance $f_{K}$ and $f_{\eta}$ are about $20 \%-$ $30 \%$ bigger than $f_{\pi}$. On the other hand, $f_{D}=1.7 f_{\pi}$ and 
$f_{D_{s}}=2.24 f_{\pi}$. So far we have taken $f_{\pi}$ for the light mesons and one value for $f_{D}=1.77 f_{\pi}$.

In view of this we shall vary these parameters in the calculation in order to estimate the uncertainties of the results

Other parameters used in the model are $m_{L}$ and $m_{H}$ which appear in the correction factors $\gamma, \psi_{3}$, and $\psi_{5}$. These parameters should be fixed by the masses of the vector mesons, the lowest possible value for the light vectors being the $\rho$ mass $(770 \mathrm{MeV})$ and the highest one the $K^{*}$ mass $(892 \mathrm{MeV})$, while for the heavy ones we have $m_{D^{*}}=2008 \mathrm{MeV}$ and $m_{D_{s}^{*}}=2112 \mathrm{MeV}$. The $J / \psi$ mass is fixed to $3097 \mathrm{MeV}$.

To study the theoretical uncertainties in our model and the stability of our results we will create random sets of values for the parameters $m_{L}, m_{H}, f_{D}, f_{\pi}$, and $\alpha_{H}$ in the proper physical allowed range. For each set $i$ of parameters we look for the poles generated and calculate their residues in the different channels. Some sets, in determinate sectors, may not generate poles, producing instead cusps close to some threshold, which will give us information about the stability of the results.

We take a range for $m_{L}, m_{H}$, and $f_{D}$ given by the average value between the magnitude of these quantities for the different mesons, plus minus the dispersion from the average, hence $m_{L} \in[745,885] \mathrm{MeV}, \quad m_{H} \in$ $[1983,2103] \mathrm{MeV}$, and $f_{D} \in[146,218] \mathrm{MeV}$. For the subtraction constant $\alpha_{H}$ we randomly choose values between -0.9 and -1.7 and for $f_{\pi}$ values between $85 \mathrm{MeV}$ and $115 \mathrm{MeV}$.

With an ensemble of 500 sets of randomly generated parameters we calculated the average pole position and the average residues in each channel in the sets where a pole was generated, and we also calculated the standard deviation from the average with:

$$
\sigma^{2}=\frac{\sum_{i=1}^{N}\left(\bar{X}-X_{i}\right)^{2}}{N-1}
$$

In Eq. (38), $\bar{X}$ is the mean value of the resonance magnitude we are calculating (pole position or residue), $X_{i}$ is the value of this magnitude for parameter set $i$ and $\mathrm{N}$ is the number of sets used for the average. This statistical study was done for the five $\mathrm{C}=1$ resonances and for the hidden charm one, since the study of these resonances is one of the main points of interest in this work.

The pole positions with uncertainties are given in Table V. In the next sections we will briefly discuss the results for each sector separately, presenting also the results for the residues of the resonances of interest.

The resonances belonging to the heavy sextet (3rd, 4th, 5th of Table V) are all stable, and a pole could be identified in all random sets. Also the $D^{*}(2400)$ pole is stable. The $D_{s}^{*}(2317)$ pole appeared for $\sim 83 \%$ of the sets; it becomes a cusp in the $D K$ threshold for the remaining sets. The
TABLE V. Pole positions with uncertainties.

\begin{tabular}{lccc|c|c}
\hline \hline Resonance ID & $\mathrm{C}$ & $\mathrm{S}$ & $\mathrm{I}$ & $\mathrm{RE}(\sqrt{s})(\mathrm{MeV})$ & $\mathrm{IM}(\sqrt{s})(\mathrm{MeV})$ \\
\hline$D_{s 0}^{*}(2317)$ & 1 & 1 & 0 & $2316 \pm 39$ & 0 \\
$D_{0}^{*}(2400)$ & 1 & 0 & $\frac{1}{2}$ & $2168 \pm 48$ & $-206 \pm 74$ \\
$(?)$ & 1 & 0 & $\frac{1}{2}$ & $2727 \pm 39$ & $-509 \pm 71$ \\
$(?)$ & 1 & 1 & 1 & $2737 \pm 40$ & $-529 \pm 70$ \\
$(?)$ & 1 & -1 & 0 & $2721 \pm 38$ & $-500 \pm 74$ \\
$(?)$ & 0 & 0 & 0 & $3698 \pm 35$ & $-0.10 \pm 0.06$ \\
\hline \hline
\end{tabular}

hidden charm resonance could be seen as a pole in $\sim 61 \%$ of the sets, appearing as a cusp over the $D \bar{D}$ threshold for the remaining ones. In Fig. 5 we show, as an example, the pole positions for each set of parameters for the $\mathrm{C}=1, \mathrm{~S}=0, \mathrm{I}=\frac{1}{2}$ sector corresponding to the $D^{*}(2400)$ resonance.

The next step was to study the effects of each one of the parameters separately over the pole positions of the resonances. In Fig. 6 one can see the effect of varying separately $f_{D}, f_{\pi}, \alpha_{H}$, and $\gamma$ over the pole position of the $D_{s}^{*}(2317)$ resonance. Except for the $\gamma$ parameter all others have a sensible effect over the pole position. Similar results are observed for its antitriplet companion, the $D^{*}(2400)$, and for the resonances belonging to the sextet. This result shows that the differences between the results of our model and previous ones $([15,17])$ in the widths of the heavy sextet resonances are not due to the exchanges of heavy vector mesons considered in this work, but rather due to the choice of a different meson decay constant for the heavy mesons and the inclusion of a term in the Lagrangian proportional to the masses, which are the other two main differences in the construction of the models.

In Figs. 7 and 8 we show similar plots for the hidden charm resonance. Here also the parameters $\alpha_{H}$ and $f_{D}$ have important effects on the mass of the resonance, but now while the parameters $f_{\pi}$ and $\gamma$ have a negligible effect

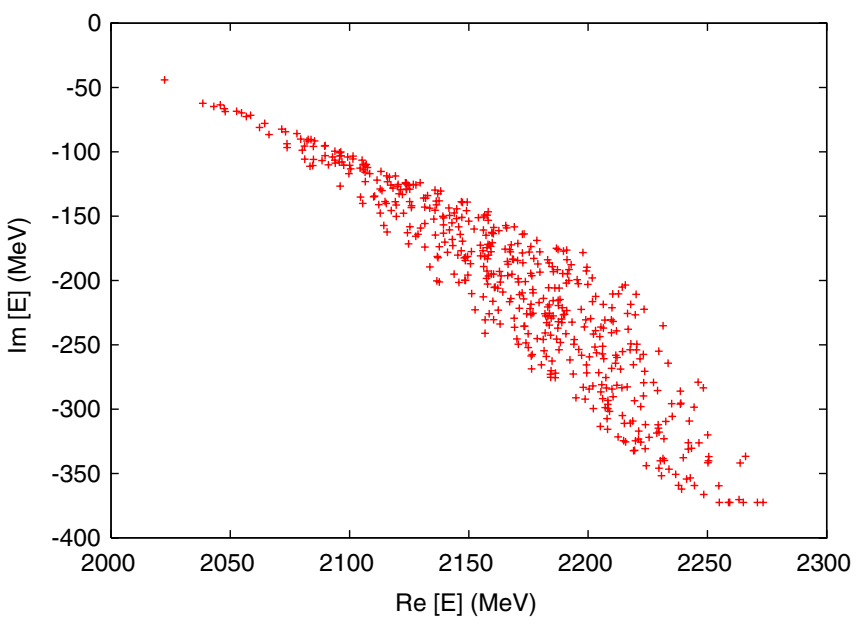

FIG. 5 (color online). Pole positions of the $D^{*}(2400)$ resonance for the different parameter sets. 

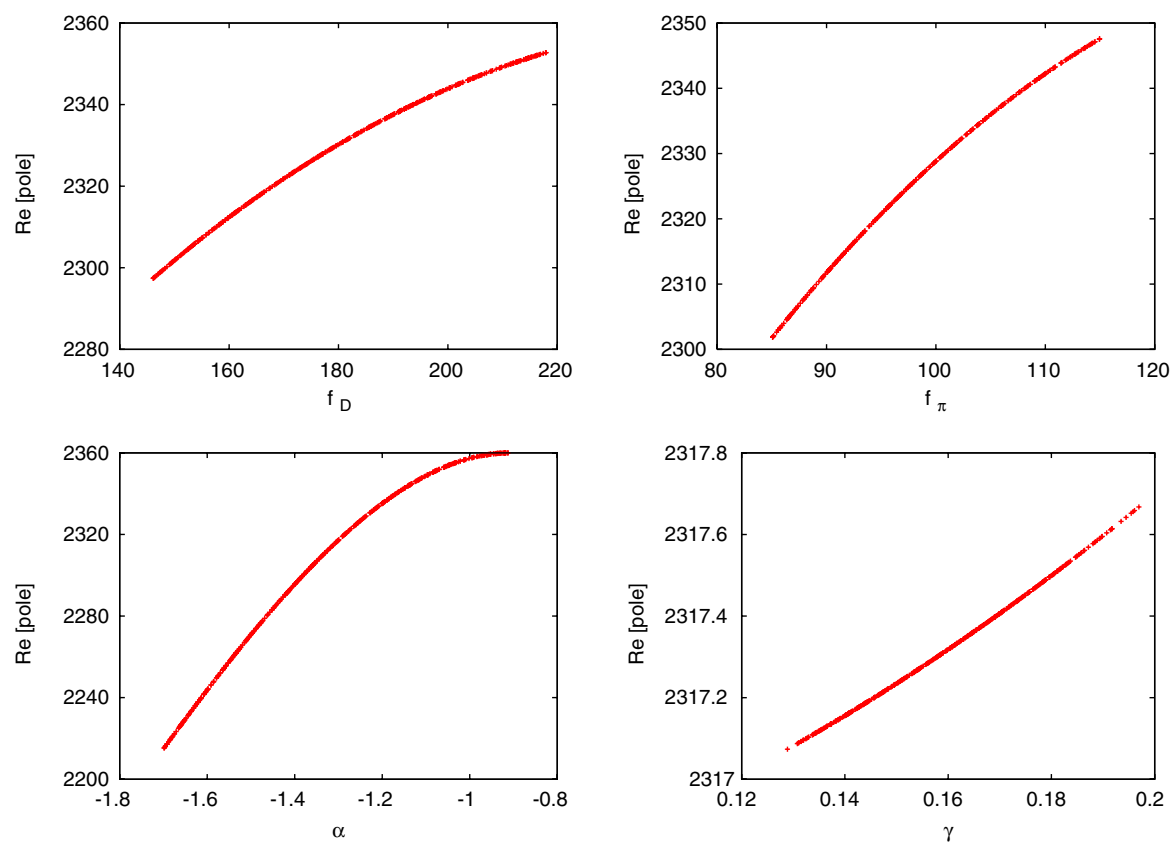

FIG. 6 (color online). Results of varying each parameter over the $D_{s}^{*}(2317)$ pole position.

over the mass of the resonance, the parameter $\gamma$ is determinant in the width of this resonance, which in any case is very small. One can see from the formalism that the processes contributing to the width are driven by the heavy vector-meson exchanges ( $\gamma$ factor), hence the sensitivity of the width to $\gamma$.

$$
\text { C. } \mathbf{C}=\mathbf{0}, \mathbf{S}=\mathbf{0}, \mathbf{I}=\mathbf{0}
$$

Our model successfully generates poles which can be associated with the known light scalar resonances. In this
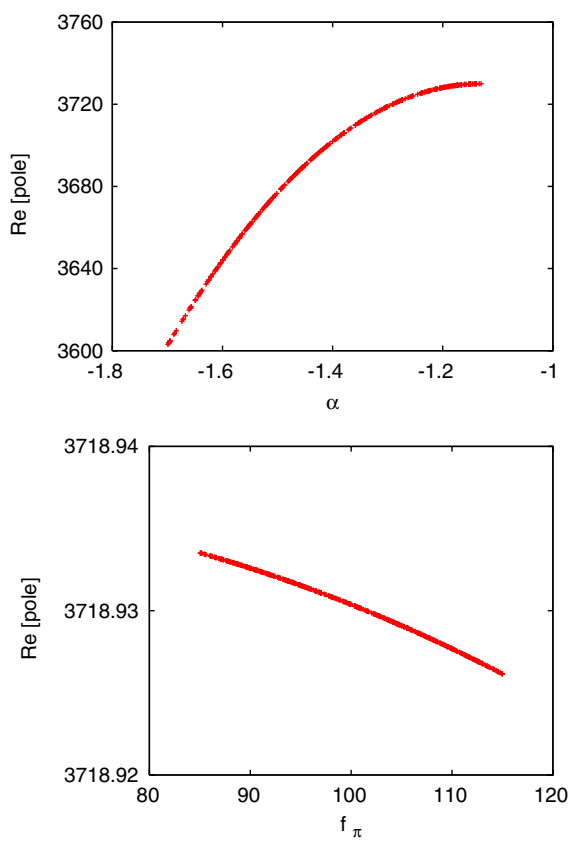

sector, in the low energy region, two poles can be found in the T-matrix, one corresponding to the $f_{0}$, but with a lower mass than one expects and another one for the $\sigma$. It is actually possible to adjust the mass of the $f_{0}$ pole in our model by increasing the $\alpha_{L}$ parameter, but two prices are paid: first the $a_{0}$ pole in the $S=0, I=1$ sector disappears for much bigger $\alpha_{L}$ and also the width of a more massive $f_{0}$ decreases.

Two more poles can be expected in this sector, one from the octet and the other one from the singlet, coming from
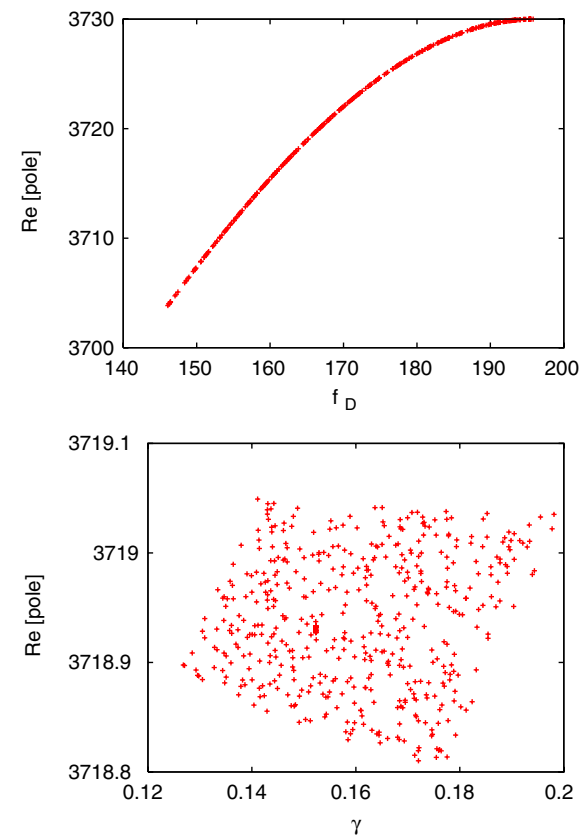

FIG. 7 (color online). Results of varying each parameter over the hidden charm resonance mass. 

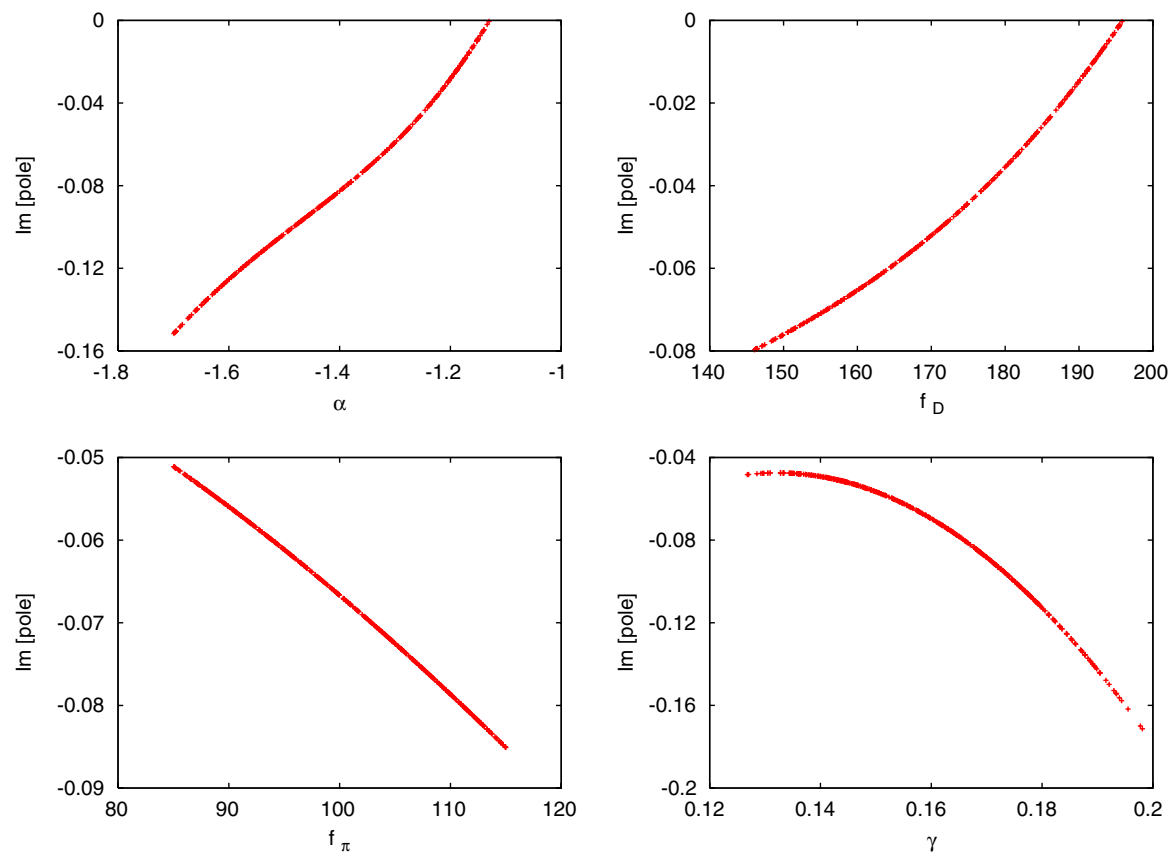

FIG. 8 (color online). Results of varying each parameter over the hidden charm resonance width.

the scattering of the heavy mesons. For $x=0$ both poles appear, the singlet always very narrow, because its coupling to the light channels is very suppressed, and the octet with a much bigger width. The octet state disappears before $x=1.0$. One should notice that the width found for this new heavy resonance is very small. This happens

TABLE VI. Residues for the poles in the $\mathrm{C}=0, \mathrm{~S}=0, \mathrm{I}=0$ sector.

\begin{tabular}{lccc}
\hline \hline Channel & $f_{0}(\mathrm{GeV})$ & $\sigma(\mathrm{GeV})$ & Heavy singlet $(\mathrm{GeV})$ \\
\hline$\pi \pi$ & 1.37 & 3.00 & $0.16 \pm 0.05$ \\
$K \bar{K}$ & 3.80 & 1.25 & $0.05 \pm 0.03$ \\
$\eta \eta$ & 3.14 & 0.36 & $0.01 \pm 0.01$ \\
$D \bar{D}$ & 0.73 & 4.14 & $11.44 \pm 4.42$ \\
$D_{s} \bar{D}_{s}$ & 3.73 & 0.49 & $7.55 \pm 2.97$ \\
$\eta \eta_{c}$ & 1.97 & 0.98 & $0.12 \pm 0.09$ \\
\hline \hline
\end{tabular}

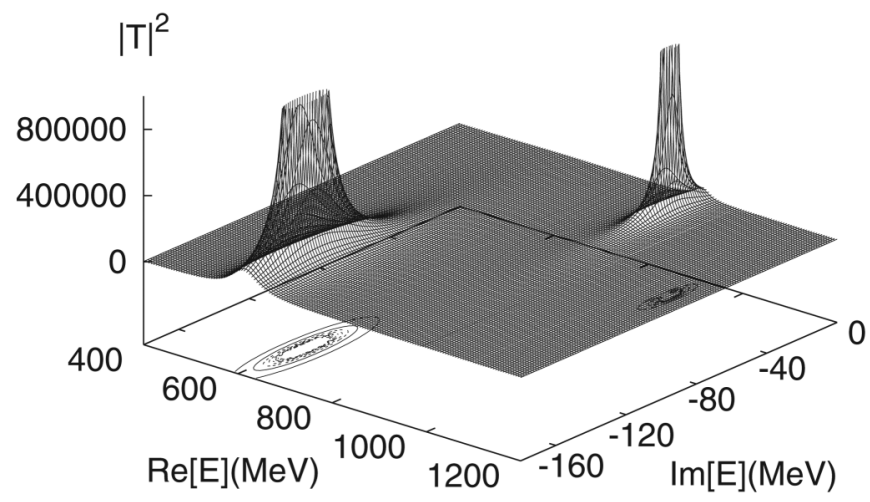

FIG. 9. $T T^{\dagger}$ for $\pi \pi$-channel in $\mathrm{C}=0, \mathrm{~S}=0, \mathrm{I}=0$ sector. because, as mentioned (see Table VI), the couplings to the light channels are very suppressed and the other possible decay channel is an octet formed by $\eta_{c}$ with a light meson which violates $S U(3)$. Table VI shows the absolute value of the residues for the resonances in this sector.

Figure 9 shows the absolute value of the square of the transition matrix for this sector, as an illustration.

$$
\text { D. } \mathrm{C}=\mathbf{0}, \mathrm{S}=\mathbf{0}, \mathrm{I}=\mathbf{1}
$$

In this sector the model successfully generates the $a_{0}$ resonance. Both the mass and the width found for it in the model agree very well with experimental values. Note, however, that this sector was actually used to fit $\alpha_{L}$, but fitting just this one parameter, both the width and the mass for the $a_{0}$ are in good agreement with experiment. As mentioned if we used the pole position of the $f_{0}$ resonance to adjust the parameter $\alpha_{L}$, we would lose the $a_{0}$ pole. This relative instability of the $a_{0}$ resonance with respect to the parameters of the theory is not new, it also occurs when using the inverse amplitude method for unitarization and the potential of the lowest order chiral Lagrangian where the $a_{0}$ appears as a cusp and not a pole. The pole is, however, regained when the information of the second order Lagrangian is used as input in the potential [23].

Table VII shows its couplings ${ }^{2}$ to the different channels.

In the heavy sector again the pole for the octet just appears for small values of $x$.

\footnotetext{
${ }^{2}$ Because of the identical particles, the $\pi \pi$ channel in $I=1$ just contributes to odd parity partial waves (indeed, the amplitude has a $p$-wave structure $t-u$ )
} 
TABLE VII. Residues for the $a_{0}$ pole.

\begin{tabular}{lc}
\hline \hline Channel & $a_{0}(\mathrm{GeV})$ \\
\hline$\pi \pi$ & 0 \\
$K \bar{K}$ & 3.84 \\
$\eta \pi$ & 2.65 \\
$D \bar{D}$ & 3.64 \\
$\pi \eta_{c}$ & 1.60 \\
\hline \hline
\end{tabular}

TABLE VIII. Residues for the $\kappa$ pole.

\begin{tabular}{lc}
\hline \hline Channel & $\kappa(\mathrm{GeV})$ \\
\hline$K \pi$ & 4.00 \\
$K \eta$ & 2.17 \\
$D_{s} \bar{D}$ & 4.12 \\
$K \eta_{c}$ & 1.88 \\
\hline \hline
\end{tabular}

$$
\text { E. } \mathrm{C}=\mathbf{0}, \mathrm{S}=\mathbf{1}, \mathrm{I}=\frac{1}{2}
$$

The pole generated here should be identified with the $\kappa$ resonance. This resonance, however, is a very broad one and although there is debate on the existence of this resonance, recent experiments have come to support it [42-47].

Again there are no heavy resonances for $x=1$.

Table VIII shows the couplings of this resonance to the various channels.

$$
\text { F. } \mathrm{C}=\mathbf{1}, \mathrm{S}=\mathbf{1}, \mathrm{I}=\mathbf{0}
$$

The $D_{s 0}^{*}(2317)$ is reproduced in this work as a mixed bound state of $|D K\rangle$ and $\left|D_{s} \eta\right\rangle$. Experimentally the observed decay channel for this resonance is $D_{s} \pi$ which is not allowed in the model because it is an isospin violating process. However if one considers isospin violation by solving the Bethe-Salpeter equation for charge eigenstates instead of isospin ones and considering the real masses of the mesons, including the differences between different $I_{3}$ components, one gets a very narrow width of less then a $\mathrm{keV}$ for this resonance. Another possible source of contribution is to consider $\eta-\pi^{0}$ mixing by means of which in [17] one gets a width of the order of a few $\mathrm{keV}$. The width of this resonance is given as an upper bound of about $4 \mathrm{MeV}$ in [35].

The couplings of this pole to the channels is shown in Table IX for both models considered in this work.

$$
\text { G. } \mathrm{C}=\mathbf{1}, \mathrm{S}=\mathbf{0}, \mathrm{I}=\frac{1}{2}
$$

Two poles are found here, one is the antitriplet companion of the $D_{s 0}^{*}(2317)$, also experimentally known and to be identified as $D_{0}^{*}(2400)$. Although the antitriplet pole generated by the model in this sector has a width in agreement with the experimental value, the model fails in predicting its mass by around $150 \mathrm{MeV}$, which might not be too
TABLE IX. Residues for the $D_{s 0}^{*}(2317)$ pole.

\begin{tabular}{lcc}
\hline \hline Channel & Chiral model $(\mathrm{GeV})$ & Phenom. model $(\mathrm{GeV})$ \\
\hline$D K$ & 10.21 & $9.08 \pm 2.53$ \\
$D_{s} \eta$ & 6.40 & $5.25 \pm 1.43$ \\
$D_{s} \eta_{c}$ & 0.48 & $1.45 \pm 0.47$ \\
\hline \hline
\end{tabular}

TABLE X. Residues for the $D_{0}^{*}(2400)$ pole.

\begin{tabular}{lcc}
\hline \hline Channel & Chiral model $(\mathrm{GeV})$ & Phenom. model $(\mathrm{GeV})$ \\
\hline$D \pi$ & 8.91 & $11.31 \pm 0.78$ \\
$D \eta$ & 1.36 & $3.46 \pm 0.27$ \\
$D \bar{s} \bar{K}$ & 5.71 & $8.58 \pm 0.32$ \\
$D \eta_{c}$ & 3.20 & $2.20 \pm 0.18$ \\
\hline \hline
\end{tabular}

serious considering that the experimental width is around $300 \mathrm{MeV}$.

Additionally another state is generated, belonging to the sextet. Here the two models differ from each other. In the chiral model this resonance has a smaller mass and width, but disappears as $x$ reaches 1 because of thresholds effects, while the pole is predicted by our model around $2700 \mathrm{MeV}$ but with a huge width that makes it irrelevant from the experimental point of view.

Residues for the $D_{0}^{*}(2400)$ pole are in Table X for both models.

H. $\mathrm{C}=1, \mathrm{~S}=1, \mathrm{I}=1$ and $\mathrm{C}=1, \mathrm{~S}=-1, \mathrm{I}=\mathbf{0}$

The other two states belonging to the sextet are to be found in these sectors. However, they differ in mass and width from one model to the other. While with the chiral Lagrangian these poles have mass around $2400 \mathrm{MeV}$ and width about $0.5 \mathrm{GeV}$, within our model their mass is $300 \mathrm{MeV}$ larger and the huge width of the order of $1 \mathrm{GeV}$ would make these poles irrelevant from the experimental point of view.

\section{Comparison with other works}

The light scalar resonances reproduced in this work have been thoroughly investigated in more sophisticated approaches and with higher orders of the chiral Lagrangian [22-24,28,29]. In our study of the hidden charm states we have now used coupled channels involving light and heavy pseudoscalar mesons and we find actually a negligible mixing of the two sectors.

The open charm sector has been studied by Kolomeitsev [15] and Guo [17] in a very similar framework but with different Lagrangians from ours; both have used the same Lagrangian, and very similar parameters. The Lagrangian in these works neglects exchange of heavy vector mesons while the present work includes it although suppressed in a proper way. In [16] higher order chiral Lagrangians are used in this sector. The second term of the Lagrangian in 
Eq. (21) can be identified with the lowest order chiral Lagrangian used in $[15,17]$ except that in the present work this term of the Lagrangian is a factor $\frac{3}{2}$ smaller. Another difference between this present work and previous ones is the meson decay constant, $f$. In previous works it was always set to the pion decay constant, while in the present one, inspired by experimental measurements and lattice calculations we use a different value for the decay constant of the charmed mesons.

In the $S=1, I=0$ sector the results of all works coincide and the $D_{s 0}^{*}(2317)$ is well reproduced. Its antitriplet companion, the $D_{0}^{*}(2400)$, is also well reproduced in the $S=0, I=\frac{1}{2}$ sector. However, in this sector the present work differs from previous ones: while within our model, the sextet state is extremely broad, in the works of Kolomeitsev and Guo a narrow state is predicted in this sector. The chiral Lagrangian we used seams to give an intermediate situation between our work and these previous ones; it generates for the sextet states a broad resonance although not as broad as in our model. The huge width of these resonances within our model is also a consequence of its much bigger mass which causes a much bigger phase-space for decay into the open channels.

Another novelty in the present work is the study of the hidden charm sector. Here we mixed light with heavy pseudoscalar pairs and concluded that there was barely any mixing of the heavy and light sectors. This result supports the findings for the light scalars, using only light pseudoscalar mesons as building blocks. On the other hand we find a heavy scalar with mass around $3.7 \mathrm{GeV}$ corresponding mostly to a $D \bar{D}$ state, very narrow (see Table IV), which is surprising in view of the large phase-space available for decay into pairs of pseudoscalars. The dynamics which prevents the mixing of the heavy and light sectors is responsible for this very small width.

We should also note that with a different formalism using the Schrödinger equation with one vector-meson exchange potential, $D \bar{D}$ states also appear for some choices of a cutoff parameter in [48].

\section{SUMMARY}

We studied the dynamical generation of resonances in a unitarized coupled channel framework. We constructed a Lagrangian based on $S U(4)$ flavor symmetry and after decomposing the field of pseudoscalar mesons in this Lagrangian into its $S U(3)$ components, we were able to identify terms mediated by exchange of heavy vector mesons and thus suppress these, hence breaking the $S U$ (4) structure of the Lagrangian. The results were also compared with previous works based on chiral theory and heavy quark symmetry and with results obtained from a chiral Lagrangian considering flavor symmetry breaking effects.
The amplitudes calculated from this Lagrangian, written in a $S U(3)$ basis, show in which sectors the interaction is attractive so that it might generate resonances. Within the framework developed in the present work a $S U(3)$ octet and a singlet of scalar mesons appear in the light sector. These resonances can be identified with the light scalar mesons, $\sigma, f_{0}, a_{0}$, and $\kappa$, which have been thoroughly investigated before, but which also show up, practically undisturbed, in the enlarged basis of coupled channels used in the present work.

In the heavy sector, an antitriplet is generated leading to two states which can be identified with the controversial $D_{s 0}^{*}(2317)$ and $D_{0}^{*}(2400)$ states, though the mass generated for this second one is somewhat lower than the experimental one. Thus, in the framework developed here, these scalar states should be interpreted as bound and quasibound states in coupled channels: The $D_{s 0}^{*}(2317)$ being mainly a $D K$ bound state with no decay, except for a tiny one when allowing isospin violation and the $D_{0}^{*}(2400)$ a $D \pi$ resonance.

Also a very broad sextet is generated in the heavy sector, but these states are extremely broad, making them irrelevant from the experimental point of view. One should note, however, that these broad states contrast with states generated in previous works $[15,17]$ where narrow structures are found with the same quantum numbers. The Lagrangian in these previous works neglects the exchange of heavy vector mesons and uses a much stronger coupling, since they use the $f_{\pi}$ parameter in all sectors, and we found that using different $f_{\pi}$ and $f_{D}$ was the main source for the large widths.

Also a heavy singlet appears as a pole in the T-matrix. This singlet comes from the attraction generated in the $\overline{3} \otimes$ 3 sector and its structure is mainly a $D \bar{D}$ quasibound state.

We also made an error analysis of the results, from where uncertainties in the results were estimated. It also served to test the stability of the results, observing if the poles disappear for some values of the parameters within the allowed range. We concluded that the uncertainties were moderate and all states were basically stable, with the exception of the hidden charm state which appeared in two thirds of the cases as a pole in the $D \bar{D}$ bound region. In one third of the cases this pole disappeared and was replaced by a cusp. Since both poles and pronounced cusps are a consequence of a strong attraction, the observation of a bound state or a strong cusp in $D \bar{D}$ would be an important finding.

\section{ACKNOWLEDGMENTS}

This work is partly supported by DGICYT Contract No. BFM2003-00856 and the Generalitat Valenciana. This research is part of the EU Integrated Infrastructure Initiative Hadron Physics Project under Contract No. RII3CT-2004-506078. 


\section{APPENDIX A: AMPLITUDES}

This appendix shows the amplitudes obtained from the Lagrangian in Eq. (28). In the column of the states, the following momenta assignments should be taken into account: where it reads $M_{1} M_{2} \rightarrow M_{3} M_{4}$ it means $M_{1}(p) M_{2}(k) \rightarrow M_{3}\left(p^{\prime}\right) M_{4}\left(k^{\prime}\right)$ and the Mandelstam variables are defined as follows:

$$
\begin{aligned}
& s=(p+k)^{2}=\left(p^{\prime}+k^{\prime}\right)^{2}, \\
& t=\left(p-p^{\prime}\right)^{2}=\left(k-k^{\prime}\right)^{2}, \\
& u=\left(p-k^{\prime}\right)^{2}=\left(k-p^{\prime}\right)^{2} .
\end{aligned}
$$

When inserting these amplitudes [or transformed to isospin or $S U(3)$ basis] in the Bethe-Salpeter equation, one should be careful to divide the amplitude by $\frac{1}{\sqrt{2}}$ each time the initial or the final state contains a pair of identical particles (unitary normalization) in order to ensure closure of the intermediate states. The extra normalization for the external lines must be kept in mind but does not matter for the pole search. The factors $\gamma, \psi_{3}$, and $\psi_{5}$ are defined in Eqs. (25)-(27).

$$
\text { 1. } \mathrm{C}=\mathbf{2}, \mathrm{S}=\mathbf{2}
$$

\begin{tabular}{lc}
\hline \hline States & Amplitude \\
\hline$D_{s}^{+} D_{s}^{+} \rightarrow D_{s}^{+} D_{s}^{+}$ & $-\frac{1}{3 f^{2}}\left(-\psi_{3}(2 s-t-u)+2 m_{D}^{2}+2 m_{K}^{2}-2 m_{\pi}^{2}\right)$ \\
\hline \hline
\end{tabular}

$$
\text { 2. } \mathrm{C}=\mathbf{2}, \mathrm{S}=\mathbf{1}
$$

\begin{tabular}{cc}
\hline \hline States & Amplitude \\
\hline$D_{s}^{+} D^{0} \rightarrow D_{s}^{+} D^{0}$ & $-\frac{1}{6 f^{2}}\left(-\psi_{5}(s-u)-(s-t)+2 m_{D}^{2}+2 m_{K}^{2}-2 m_{\pi}^{2}\right)$ \\
\hline \hline
\end{tabular}

$$
\text { 3. } \mathrm{C}=\mathbf{2}, \mathrm{S}=\mathbf{0}
$$

\begin{tabular}{|c|c|}
\hline States & Amplitude \\
\hline $\begin{aligned} K^{+} D^{0} & \rightarrow K^{+} D^{0} \\
& \rightarrow K^{0} D^{+} \\
& \rightarrow \pi^{0} D_{s}^{+} \\
& \rightarrow \eta D_{s}^{+}\end{aligned}$ & $\begin{array}{l}-\frac{1}{6 f^{2}}\left(\gamma(t-u)+(s-u)+m_{D}^{2}+m_{K}^{2}\right) \\
-\frac{1}{6 f^{2}}\left(\gamma(t-u)+(s-u)+m_{D}^{2}+m_{K}^{2}\right) \\
-\frac{1}{6 \sqrt{2} f^{2}}\left(-(s-u)-\gamma(s-t)+m_{D}^{2}+m_{K}^{2}\right) \\
-\frac{1}{6 \sqrt{6} f^{2}}\left(\gamma(u-t)-(3+\gamma)(s-u)-m_{D}^{2}\right. \\
\left.\quad-3 m_{K}^{2}+2 m_{\pi}^{2}\right)\end{array}$ \\
\hline $\begin{aligned} K^{0} D^{+} & \rightarrow K^{0} D^{+} \\
& \rightarrow \pi^{0} D_{s}^{+} \\
& \rightarrow \eta D_{s}^{+}\end{aligned}$ & $\begin{array}{l}-\frac{1}{6 f^{2}}\left(\gamma(t-u)+(s-u)+m_{D}^{2}+m_{K}^{2}\right) \\
-\frac{1}{6 \sqrt{2} f^{2}}\left((s-u)+\gamma(s-t)-m_{D}^{2}-m_{K}^{2}\right) \\
-\frac{1}{6 \sqrt{6} f^{2}}(\gamma(u-t)-(3+\gamma)(s-u) \\
\left.\quad-m_{D}^{2}-3 m_{K}^{2}+2 m_{\pi}^{2}\right)\end{array}$ \\
\hline $\begin{aligned} \pi^{0} D_{s}^{+} & \rightarrow \pi^{0} D_{s}^{+} \\
& \rightarrow \eta D_{s}^{+} \\
\eta D_{s}^{+} & \rightarrow \eta D_{s}^{+}\end{aligned}$ & $\begin{aligned}-\frac{1}{9 f^{2}}(\gamma(-s+2 t-u) & \left.+2 m_{D}^{2}+6 m_{K}^{2}-4 m_{\pi}^{2}\right)\end{aligned}$ \\
\hline $\begin{aligned} \eta_{c} D_{s}^{+} & \rightarrow \eta_{c} D_{s}^{+} \\
& \rightarrow K^{+} D^{0} \\
& \rightarrow K^{0} D^{+} \\
& \rightarrow \pi^{0} D_{s}^{+} \\
& \rightarrow \eta D_{s}^{+}\end{aligned}$ & $\begin{array}{l}-\frac{1}{18 f^{2}}\left(4 \gamma(-s+2 t-u)+11 m_{D}^{2}\right. \\
\left.\quad+3 m_{K}^{2}-7 m_{\pi}^{2}\right) \\
-\frac{1}{6 \sqrt{3} f^{2}}\left(2 \gamma(-s+2 t-u)-2 m_{D}^{2}+m_{\pi}^{2}\right) \\
-\frac{1}{6 \sqrt{3} f^{2}}\left(2 \gamma(-s+2 t-u)-2 m_{D}^{2}+m_{\pi}^{2}\right) \\
- \\
-\frac{1}{9 \sqrt{2} f^{2}}\left(-2 \gamma(-s+2 t-u)+2 m_{D}^{2}-m_{\pi}^{2}\right)\end{array}$ \\
\hline
\end{tabular}

\begin{tabular}{lc}
\hline \hline States & Amplitude \\
\hline$D^{+} D^{0} \rightarrow D^{+} D^{0}$ & $-\frac{1}{6 f^{2}}\left(-\psi_{5}(s-u)-(s-t)+2 m_{D}^{2}\right)$ \\
\hline \hline
\end{tabular}

\begin{tabular}{|c|c|}
\hline States & Amplitude \\
\hline$\pi^{0} D^{0} \rightarrow \pi^{0} D^{0}$ & $-\frac{1}{12 f^{2}}\left(\gamma(-s+2 t-u)+2 m_{D}^{2}+2 m_{\pi}^{2}\right)$ \\
\hline$\rightarrow \pi^{-} D^{+}$ & $-\frac{1}{6 \sqrt{2} f^{2}}((2+\gamma)(s-u))$ \\
\hline$\rightarrow \eta D^{0}$ & $-\frac{1}{12 \sqrt{3} f^{2}}\left(\gamma(-s+2 t-u)+2 m_{D}^{2}+2 m_{\pi}^{2}\right)$ \\
\hline$\rightarrow K^{-} D_{s}^{+}$ & $-\frac{1}{6 \sqrt{2} f^{2}}\left(\gamma(t-u)+s-u+m_{D}^{2}+m_{K}^{2}\right)$ \\
\hline$\pi^{-} D^{+} \rightarrow \pi^{-} D^{+}$ & $-\frac{1}{6 f^{2}}\left(\gamma(t-u)+s-u+m_{D}^{2}+m_{\pi}^{2}\right)$ \\
\hline$\rightarrow \eta D^{0}$ & $-\frac{1}{6 \sqrt{6} f^{2}}\left(\gamma(-s+2 t-u)+2 m_{D}^{2}+2 m_{\pi}^{2}\right)$ \\
\hline$\rightarrow K^{-} D_{s}^{+}$ & $-\frac{1}{6 f^{2}}\left(\gamma(t-u)+s-u+m_{D}^{2}+m_{K}^{2}\right)$ \\
\hline$\eta D^{0} \rightarrow \eta D^{0}$ & $-\frac{1}{36 f^{2}}\left(\gamma(-s+2 t-u)+2 m_{D}^{2}+2 m_{\pi}^{2}\right)$ \\
\hline$\rightarrow K^{-} D_{s}^{+}$ & $\begin{array}{l}-\frac{1}{6 \sqrt{6} f^{2}}\left(\gamma(s-t)+(3+\gamma)(s-u)-m_{D}^{2}\right. \\
\left.\quad 3 m_{K}^{2}+2 m_{\pi}^{2}\right)\end{array}$ \\
\hline$K^{-} D_{s}^{+} \rightarrow K^{-} D_{s}^{+}$ & $-\frac{1}{6 f^{2}}\left(\gamma(t-u)+s-u+m_{D}^{2}+2 m_{K}^{2}-m_{\pi}^{2}\right)$ \\
\hline$\eta_{c} D^{0} \rightarrow \eta_{c} D^{0}$ & $-\frac{1}{18 f^{2}}\left(4 \gamma(-s+2 t-u)+11 m_{D}^{2}-4 m_{\pi}^{2}\right)$ \\
\hline$\rightarrow \pi^{0} D^{0}$ & $-\frac{1}{6 \sqrt{6} f^{2}}\left(2 \gamma(-s+2 t-u)-2 m_{D}^{2}+m_{\pi}^{2}\right)$ \\
\hline$\rightarrow \pi^{-} D^{+}$ & $-\frac{1}{6 \sqrt{3} f^{2}}\left(2 \gamma(-s+2 t-u)-2 m_{D}^{2}+m_{\pi}^{2}\right)$ \\
\hline$\rightarrow \eta D^{0}$ & $-\frac{1}{18 \sqrt{2} f^{2}}\left(2 \gamma(-s+2 t-u)-2 m_{D}^{2}+m_{\pi}^{2}\right)$ \\
\hline$\rightarrow K^{-} D_{s}^{+}$ & $-\frac{1}{6 \sqrt{3} f^{2}}\left(2 \gamma(-s+2 t-u)-2 m_{D}^{2}+m_{\pi}^{2}\right)$ \\
\hline
\end{tabular}

4. $\mathrm{C}=1, \mathrm{~S}=2$

\begin{tabular}{lc}
\hline \hline States & Amplitude \\
\hline$K^{0} D_{s}^{+} \rightarrow K^{0} D_{s}^{+}$ & $-\frac{1}{6 f^{2}}\left(-(s-u)-\gamma(s-t)+m_{D}^{2}+2 m_{K}^{2}-m_{\pi}^{2}\right)$ \\
\hline \hline
\end{tabular}

\begin{tabular}{|c|c|}
\hline States & Amplitude \\
\hline $\begin{aligned} K^{-} D^{+} & \rightarrow K^{-} D^{+} \\
& \rightarrow \bar{K}^{0} D^{0}\end{aligned}$ & $-\frac{1}{6 f^{2}}\left(-(s-u)-\gamma(s-t)+m_{D}^{2}+m_{K}^{2}\right)$ \\
\hline
\end{tabular}

5. $\mathrm{C}=1, \mathrm{~S}=1$

6. $C=1, S=0$

7. $\mathrm{C}=1, \mathrm{~S}=-1$ 


\begin{tabular}{cc} 
States & Amplitude \\
\hline $\bar{K}^{0} D^{0} \rightarrow \bar{K}^{0} D^{0}$ & - \\
\hline
\end{tabular}

\section{8. $\mathrm{C}=\mathbf{0}, \mathrm{S}=\mathbf{1}$}

\begin{aligned} & \hline \hline States \multicolumn{1}{c}{ Amplitude } \\ & \hline$D_{s}^{+} D^{-} \rightarrow D_{s}^{+} D^{-}-\frac{1}{6 f^{2}}\left(t-u+\psi 5(s-u)+2 m_{D}^{2}+m_{K}^{2}-m_{\pi}^{2}\right) \\ & \pi^{0} K^{0} \rightarrow \pi^{0} K^{0}-\frac{1}{12 f^{2}}\left(-s+2 t-u+2 m_{K}^{2}+2 m_{\pi}^{2}\right) \\ & \rightarrow \pi^{-} K^{+}-\frac{1}{2 \sqrt{2} f^{2}}(-s+u) \\ & \rightarrow \eta K^{0}-\frac{1}{12 \sqrt{3} f^{2}}\left(3(s-2 t+u)+2 m_{K}^{2}-2 m_{\pi}^{2}\right) \\ & \pi^{-} K^{+} \rightarrow \pi^{-} K^{+}-\frac{1}{6 f^{2}}\left(s+t-2 u+m_{K}^{2}+m_{\pi}^{2}\right) \\ & \rightarrow \eta K^{0}-\frac{1}{6 \sqrt{6} f^{2}}\left(-3(s-2 t+u)+6 m_{K}^{2}-2 m_{\pi}^{2}\right) \\ & \eta K^{0} \rightarrow \eta K^{0}-\frac{1}{12 f^{2}}\left(-3(s-2 t+u)+6 m_{K}^{2}-2 m_{\pi}^{2}\right) \\ & \eta_{c} K^{0} \rightarrow \eta_{c} K^{0}-\frac{1}{6 f^{2}} m_{K}^{2} \\ & D_{s}^{+} D^{-} \rightarrow \pi^{0} K^{0}-\frac{1}{6 \sqrt{2} f^{2}}\left(t-u-\gamma(s-t)-m_{D}^{2}-m_{K}^{2}\right) \\ & \rightarrow \pi^{-} K^{+}-\frac{1}{6 f^{2}}\left(-(t-u)+\gamma(s-t)+m_{D}^{2}+m_{K}^{2}\right) \\ & \rightarrow \eta K^{0}-\frac{1}{6 \sqrt{6} f^{2}}\left((3+\gamma)(u-t)-\gamma(s-u)-m_{D}^{2}\right. \\ & D_{s}^{+} D^{-} \rightarrow \eta_{c} K^{0}-\frac{1}{6 \sqrt{3} f^{2}}\left(2 \gamma(2 s-t-u)-2 m_{D}^{2}+m_{\pi}^{2}\right) \\ & \eta_{c} K^{0} \rightarrow \pi^{0} K^{0}-\frac{1}{6 \sqrt{6} f^{2}}\left(-2 m_{K}^{2}-m_{\pi}^{2}\right) \\ & \rightarrow \pi^{-} K^{+}-\frac{1}{6 \sqrt{3} f^{2}}\left(2 m_{K}^{2}+m_{\pi}^{2}\right) \\ & \rightarrow \eta K^{0}-\frac{1}{6 \sqrt{2} f^{2}}\left(-2 m_{K}^{2}+m_{\pi}^{2}\right) \\ &$\hline \hline\end{aligned}

9. $\mathbf{C}=\mathbf{0}, \mathbf{S}=\mathbf{0}$
States

Amplitude

\begin{tabular}{|c|c|}
\hline$\rightarrow \pi^{0} \eta$ & - \\
\hline$\rightarrow \eta \eta$ & $-\frac{1}{3 f^{2}} m_{\pi}^{2}$ \\
\hline$\pi^{0} \pi^{0} \rightarrow \pi^{0} \pi^{0}$ & $-\frac{1}{f^{2}} m_{\pi}^{2}$ \\
\hline$\rightarrow \pi^{0} \eta$ & - \\
\hline$\rightarrow \eta \eta$ & $-\frac{1}{3 f^{2}} m_{\pi}^{2}$ \\
\hline$\pi^{0} \eta \rightarrow \pi^{0} \eta$ & $-\frac{1}{3 f^{2}} m_{\pi}^{2}$ \\
\hline$\rightarrow \eta \eta$ & - \\
\hline$\eta \eta \rightarrow \eta \eta$ & $-\frac{1}{9 f^{2}}\left(16 m_{k}^{2}-7 m_{\pi}^{2}\right)$ \\
\hline$\eta_{c} \pi^{0} \rightarrow \eta_{c} \pi^{0}$ & $-\frac{1}{6 f^{2}} m_{\pi}^{2}$ \\
\hline$\rightarrow \eta_{c} \eta$ & - \\
\hline$\eta_{c} \eta \rightarrow \eta_{c} \eta$ & $-\frac{1}{18 f^{2}}\left(4 m_{k}^{2}-m_{\pi}^{2}\right)$ \\
\hline$D_{s}^{+} D_{s}^{-} \rightarrow K^{+} K^{-}$ & $-\frac{1}{6 f^{2}}\left(t-u+\gamma(s-u)+m_{D}^{2}+2 m_{K}^{2}-m_{\pi}^{2}\right)$ \\
\hline$\rightarrow K^{0} \bar{K}^{0}$ & $-\frac{1}{6 f^{2}}\left(t-u+\gamma(s-u)+m_{D}^{2}+2 m_{K}^{2}-m_{\pi}^{2}\right)$ \\
\hline$\rightarrow \pi^{+} \pi^{-}$ & - \\
\hline$\rightarrow \pi^{0} \pi^{0}$ & - \\
\hline$\rightarrow \pi^{0} \eta$ & - \\
\hline$\rightarrow \eta \eta$ & $-\frac{1}{9 f^{2}}\left(\gamma(2 s-t-u)+2 m_{D}^{2}+6 m_{K}^{2}-4 m_{\pi}^{2}\right)$ \\
\hline
\end{tabular}

\begin{tabular}{|c|c|}
\hline States & Amplitude \\
\hline$D^{+} D^{-} \rightarrow K^{+} K^{-}$ & - \\
\hline$\rightarrow K^{0} \bar{K}^{0}$ & $-\frac{1}{6 f^{2}}\left(-(t-u)+\gamma(s-t)+m_{D}^{2}+m_{K}^{2}\right)$ \\
\hline$\rightarrow \pi^{+} \pi^{-}$ & $-\frac{1}{6 f^{2}}\left(t-u+\gamma(s-u)+m_{D}^{2}+m_{\pi}^{2}\right)$ \\
\hline$\rightarrow \pi^{0} \pi^{0}$ & $-\frac{1}{12 f^{2}}\left(\gamma(2 s-t-u)+2 m_{D}^{2}+2 m_{\pi}^{2}\right)$ \\
\hline$\rightarrow \pi^{0} \eta$ & $-\frac{1}{12 \sqrt{3} f^{2}}\left(-\gamma(2 s-t-u)-2 m_{D}^{2}-2 m_{\pi}^{2}\right)$ \\
\hline$\rightarrow \eta \eta$ & $-\frac{1}{36 f^{2}}\left(\gamma(2 s-t-u)+2 m_{D}^{2}+2 m_{\pi}^{2}\right)$ \\
\hline$D^{0} D^{0} \rightarrow K^{+} K^{-}$ & $-\frac{1}{6 f^{2}}\left(-(t-u)+\gamma(s-t)+m_{D}^{2}+m_{K}^{2}\right)$ \\
\hline$\rightarrow K^{0} \bar{K}^{0}$ & - \\
\hline$\rightarrow \pi^{+} \pi^{-}$ & $-\frac{1}{6 f^{2}}\left(-(t-u)+\gamma(s-t)+m_{D}^{2}+m_{\pi}^{2}\right)$ \\
\hline$\rightarrow \pi^{0} \pi^{0}$ & $-\frac{1}{12 f^{2}}\left(\gamma(2 s-t-u)+2 m_{D}^{2}+2 m_{\pi}^{2}\right)$ \\
\hline$\rightarrow \pi^{0} \eta$ & $-\frac{1}{12 \sqrt{3} f^{2}}\left(\gamma(2 s-t-u)+2 m_{D}^{2}+2 m_{\pi}^{2}\right)$ \\
\hline$\rightarrow \eta \eta$ & $-\frac{1}{36 f^{2}}\left(\gamma(2 s-t-u)+2 m_{D}^{2}+2 m_{\pi}^{2}\right)$ \\
\hline$D_{s}^{+} D_{s}^{-} \rightarrow \eta_{c} \pi^{0}$ & - \\
\hline$\rightarrow \eta_{c} \eta$ & $-\frac{1}{9 \sqrt{2} f^{2}}\left(-2 \gamma(2 s-t-u)+2 m_{D}^{2}-m_{\pi}^{2}\right)$ \\
\hline$D^{+} D^{-} \rightarrow \eta_{c} \pi^{0}$ & $-\frac{1}{6 \sqrt{6} f^{2}}\left(-2 \gamma(2 s-t-u)+2 m_{D}^{2}-m_{\pi}^{2}\right)$ \\
\hline$\rightarrow \eta_{c} \eta$ & $-\frac{1}{18 \sqrt{2} f^{2}}\left(2 \gamma(2 s-t-u)-2 m_{D}^{2}+m_{\pi}^{2}\right)$ \\
\hline$D^{0} \bar{D}^{0} \rightarrow \eta_{c} \pi^{0}$ & $-\frac{1}{6 \sqrt{6} f^{2}}\left(2 \gamma(2 s-t-u)-2 m_{D}^{2}+m_{\pi}^{2}\right)$ \\
\hline$\rightarrow \eta_{c} \eta$ & $-\frac{1}{18 \sqrt{2} f^{2}}\left(2 \gamma(2 s-t-u)-2 m_{D}^{2}+m_{\pi}^{2}\right)$ \\
\hline$\eta_{c} \pi^{0} \rightarrow K^{+} K^{-}$ & $-\frac{1}{6 \sqrt{6} f^{2}}\left(2 m_{K}^{2}+m_{\pi}^{2}\right)$ \\
\hline$\rightarrow K^{0} \bar{K}^{0}$ & $-\frac{1}{6 \sqrt{6} f^{2}}\left(-2 m_{K}^{2}+m_{\pi}^{2}\right)$ \\
\hline$\rightarrow \pi^{+} \pi^{-}$ & - \\
\hline$\rightarrow \pi^{0} \pi^{0}$ & - \\
\hline$\rightarrow \pi^{0} \eta$ & $-\frac{1}{3 \sqrt{2} f^{2}} m_{\pi}^{2}$ \\
\hline$\rightarrow \eta \eta$ & - \\
\hline$\eta_{c} \eta \rightarrow K^{+} K^{-}$ & $-\frac{1}{6 \sqrt{2} f^{2}}\left(-2 m_{K}^{2}+m_{\pi}^{2}\right)$ \\
\hline
\end{tabular}




\begin{tabular}{ll} 
States & \multicolumn{1}{c}{ Amplitude } \\
\hline$\rightarrow K^{0} \bar{K}^{0}$ & $-\frac{1}{6 \sqrt{2} f^{2}}\left(-2 m_{K}^{2}+m_{\pi}^{2}\right)$ \\
$\rightarrow \pi^{+} \pi^{-}$ & $-\frac{1}{3 \sqrt{2} f^{2}} m_{\pi}^{2}$ \\
$\rightarrow \pi^{0} \pi^{0}$ & $-\frac{1}{3 \sqrt{2} f^{2}} m_{\pi}^{2}$ \\
$\rightarrow \pi^{0} \eta$ & - \\
$\rightarrow \eta \eta$ & $-\frac{1}{9 \sqrt{2} f^{2}}\left(-8 m_{K}^{2}+5 m_{\pi}^{2}\right)$ \\
\hline \hline
\end{tabular}

\section{APPENDIX B: ISOSPIN AND $S U(3)$ BASIS}

The following phases are taken for the meson assignments of the 15-plet:

$$
\begin{gathered}
\left|D_{s}\right\rangle_{0}=\left|D_{s}^{+}\right\rangle, \quad|D\rangle_{1 / 2}=\left(\begin{array}{c}
\left|D^{+}\right\rangle \\
-\left|D^{0}\right\rangle
\end{array}\right), \\
|K\rangle_{1 / 2}=\left(\begin{array}{c}
\left|K^{+}\right\rangle \\
\left|K^{0}\right\rangle
\end{array}\right), \quad|\pi\rangle_{1}=\left(\begin{array}{c}
-\left|\pi^{+}\right\rangle \\
\left|\pi^{0}\right\rangle \\
\left|\pi^{-}\right\rangle
\end{array}\right), \\
|\eta\rangle_{0}=|\eta\rangle, \quad\left|\eta_{c}\right\rangle_{0}=\left|\eta_{c}\right\rangle, \quad|\bar{K}\rangle_{1 / 2}=\left(\begin{array}{c}
\left|\bar{K}^{0}\right\rangle \\
-\left|K^{-}\right\rangle
\end{array}\right), \\
|\bar{D}\rangle_{1 / 2}=\left(\begin{array}{c}
\left|\bar{D}^{0}\right\rangle \\
\left|D^{-}\right\rangle
\end{array}\right) \quad \text { and } \quad\left|\bar{D}_{s}\right\rangle_{0}=\left|D_{s}^{-}\right\rangle .
\end{gathered}
$$

In the following we will list for the sectors where a $S U(3)$ decomposition is not trivial, the isospin and $S U(3)$ states used to transform the amplitudes from a charge basis to isospin and then from isospin into a $S U(3)$ basis. For reviews on phase conventions and isoscalar factors of the $S U$ (3) Clebsch-Gordan coefficients one can refer to [49,50].

Figures 10-13 show a pictorial representation of the multiplet multiplications.

$$
\begin{aligned}
& \text { 1. } \overline{\mathbf{3}} \otimes \overline{\mathbf{3}}(\mathrm{C}=\mathbf{2}) \\
& \left|D_{s} D_{s}\right\rangle_{0}=\left|D_{s}^{+} D_{s}^{+}\right\rangle
\end{aligned}
$$
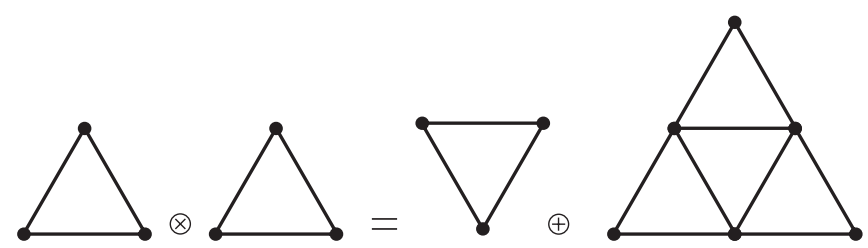

FIG. $10 . \quad \overline{3} \otimes \overline{3}=3 \oplus \overline{6}$.
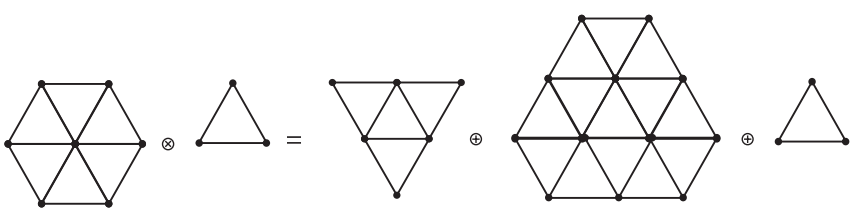

FIG. $11 . \quad 8 \otimes \overline{3}=6 \oplus \overline{15} \oplus \overline{3}$.
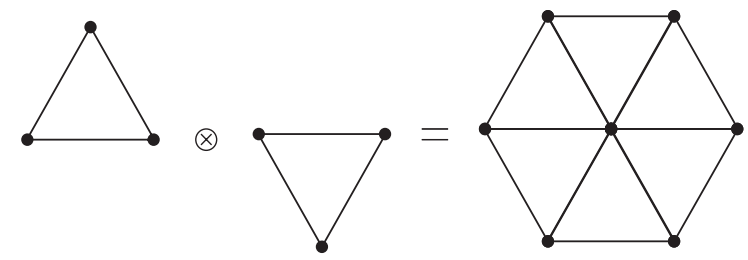

FIG. $12 . \quad \overline{3} \otimes 3=8 \oplus 1$.

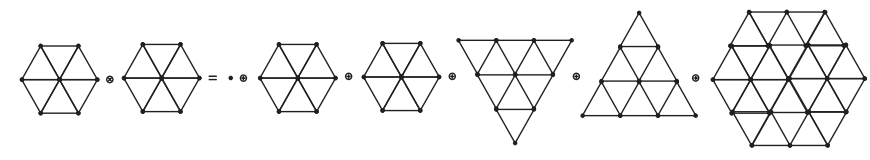

FIG. 13. $8 \otimes 8=1 \oplus 8 \oplus 8 \oplus 10 \oplus \overline{10} \oplus 27$.

$$
\begin{aligned}
& \left|D D_{s}\right\rangle_{1 / 2}=-\left|D^{0} D_{s}^{+}\right\rangle \\
& \left(\begin{array}{c}
|D D\rangle_{0} \\
|D D\rangle_{1}
\end{array}\right)=\frac{1}{\sqrt{2}}\left(\begin{array}{cc}
-1 & 1 \\
-1 & -1
\end{array}\right)\left(\begin{array}{c}
\left|D^{+} D^{0}\right\rangle \\
\left|D^{0} D^{+}\right\rangle
\end{array}\right)
\end{aligned}
$$

$|\hat{6}, 2,0\rangle=\left|D_{s} D_{s}\right\rangle \quad[S U(3)$ states are represented as $\mid$ Irrep, $S, I\rangle$.]

$\left|\overline{6}, 1, \frac{1}{2}\right\rangle=\frac{1}{\sqrt{2}}\left(\left|D D_{s}\right\rangle+\left|D_{s} D\right\rangle\right)$ [From now on the label for the isospin of the states will be omitted for the $S U(3)$ states.]

$$
\begin{aligned}
& |\overline{6}, 0,1\rangle=|D D\rangle \\
& \left|3,1, \frac{1}{2}\right\rangle=\frac{1}{\sqrt{2}}\left(\left|D D_{s}\right\rangle-\left|D_{s} D\right\rangle\right) \\
& |3,0,0\rangle=|D D\rangle
\end{aligned}
$$

\section{2. $\overline{3} \otimes 8 \mathrm{C}=1$}

$\left|K D_{s}\right\rangle_{1 / 2}=\left|K^{0} D_{s}^{+}\right\rangle$

$\left(\begin{array}{c}|K D\rangle_{0} \\ |K D\rangle_{1}\end{array}\right)=\frac{1}{\sqrt{2}}\left(\begin{array}{cc}-1 & -1 \\ -1 & 1\end{array}\right)\left(\begin{array}{l}\left|K^{+} D^{0}\right\rangle \\ \left|K^{0} D^{+}\right\rangle\end{array}\right)$

$\left|\eta D_{s}\right\rangle_{0}=\left|\eta D_{s}^{+}\right\rangle$

$\left|\pi D_{s}\right\rangle_{1}=\left|\pi^{-} D_{s}^{+}\right\rangle$

$\left(\begin{array}{l}|\pi D\rangle_{1 / 2} \\ |\pi D\rangle_{3 / 2}\end{array}\right)=\left(\begin{array}{cc}\frac{-1}{\sqrt{3}} & -\sqrt{\frac{2}{3}} \\ -\sqrt{\frac{2}{3}} & \frac{1}{\sqrt{3}}\end{array}\right)\left(\begin{array}{c}\left|\pi^{0} D^{0}\right\rangle \\ \left|\pi^{-} D^{+}\right\rangle\end{array}\right)$

$|\eta D\rangle_{1 / 2}=-\left|\eta D^{0}\right\rangle$

$\left|\bar{K} D_{s}\right\rangle_{1 / 2}=-\left|K^{-} D_{s}^{+}\right\rangle$

$\left(\begin{array}{c}|\bar{K} D\rangle_{0} \\ |\bar{K} D\rangle_{1}\end{array}\right)=\frac{1}{\sqrt{2}}\left(\begin{array}{cc}1 & -1 \\ -1 & -1\end{array}\right)\left(\begin{array}{c}\left|K^{-} D^{+}\right\rangle \\ \left|\bar{K}^{0} D^{0}\right\rangle\end{array}\right)$

$\left|\overline{15}, 2, \frac{1}{2}\right\rangle=\left|K D_{s}\right\rangle$

$|\overline{15}, 1,1\rangle=\frac{1}{\sqrt{2}}\left(|K D\rangle-\left|\pi D_{s}\right\rangle\right)$

$|\overline{15}, 1,0\rangle=-\frac{\sqrt{3}}{2}\left|\eta D_{s}\right\rangle+\frac{1}{2}|K D\rangle$

$\left|\overline{15}, 0, \frac{3}{2}\right\rangle=|\pi D\rangle$

$\left|\overline{15}, 0, \frac{1}{2}\right\rangle=\frac{1}{4}|\pi D\rangle+\frac{3}{4}|\eta D\rangle-\sqrt{\frac{3}{8}}\left|\bar{K} D_{s}\right\rangle$

$|\overline{15},-1,1\rangle=|\bar{K} D\rangle$

$|6,1,1\rangle=\frac{1}{\sqrt{2}}\left(|K D\rangle+\left|\pi D_{s}\right\rangle\right)$

$\left|6,0, \frac{1}{2}\right\rangle=\sqrt{\frac{3}{8}}|\pi D\rangle-\sqrt{\frac{3}{8}}|\eta D\rangle-\frac{1}{2}\left|\bar{K} D_{s}\right\rangle$

$|6,-1,0\rangle=|\bar{K} D\rangle$ 


$$
\begin{aligned}
& |\overline{3}, 1,0\rangle=\frac{1}{2}\left|\eta D_{s}\right\rangle+\frac{\sqrt{3}}{2}|K D\rangle \\
& \left|\overline{3}, 0, \frac{1}{2}\right\rangle=-\frac{3}{4}|\pi D\rangle-\frac{1}{4}|\eta D\rangle-\sqrt{\frac{3}{8}}\left|\bar{K} D_{s}\right\rangle
\end{aligned}
$$

\section{3. $\overline{\mathbf{3}} \otimes \mathbf{3} \mathrm{C}=\mathbf{0}$}

$\left|D_{s} \bar{D}\right\rangle_{1 / 2}=\left|D_{s}^{+} D^{-}\right\rangle$

$\left|D_{s} \bar{D}_{s}\right\rangle_{0}=\left|D_{s}^{+} D_{s}^{-}\right\rangle$

$\left(\begin{array}{l}|D \bar{D}\rangle_{0} \\ |D \bar{D}\rangle_{1}\end{array}\right)=\frac{1}{\sqrt{2}}\left(\begin{array}{cc}1 & 1 \\ 1 & -1\end{array}\right)\left(\begin{array}{c}\left|D^{+} D^{-}\right\rangle \\ \left|D^{0} \bar{D}^{0}\right\rangle\end{array}\right)$

$\left|8,1, \frac{1}{2}\right\rangle=\left|D_{s} \bar{D}\right\rangle$

$|8,0,1\rangle=|D \bar{D}\rangle$

$|8,0,0\rangle=\sqrt{\frac{2}{3}}\left|D_{s} \bar{D}_{s}\right\rangle-\frac{1}{\sqrt{3}}|D \bar{D}\rangle$

$\left|8,-1, \frac{1}{2}\right\rangle=\left|\bar{D}_{s} D\right\rangle$

$|1,0,0\rangle=\frac{1}{\sqrt{3}}\left|D_{s} \bar{D}_{s}\right\rangle+\sqrt{\frac{2}{3}}|D \bar{D}\rangle$

\section{4. $8 \otimes 8 \mathrm{C}=0$}

$\left(\begin{array}{l}|\pi K\rangle_{1 / 2} \\ |\pi K\rangle_{3 / 2}\end{array}\right)=\left(\begin{array}{cc}\frac{1}{\sqrt{3}} & -\sqrt{\frac{2}{3}} \\ \sqrt{\frac{2}{3}} & \frac{1}{\sqrt{3}}\end{array}\right)\left(\begin{array}{c}\left|\pi^{0} K^{0}\right\rangle \\ \left|\pi^{-} K^{+}\right\rangle\end{array}\right)$

$|\eta K\rangle_{1 / 2}=\left|\eta K^{0}\right\rangle$

$\left(\begin{array}{c}|K \bar{K}\rangle_{0} \\ |K \bar{K}\rangle_{1}\end{array}\right)=\frac{1}{\sqrt{2}}\left(\begin{array}{cc}-1 & -1 \\ -1 & 1\end{array}\right)\left(\begin{array}{c}\left|K^{+} K^{-}\right\rangle \\ \left|K^{0} \bar{K}^{0}\right\rangle\end{array}\right)$

$\left(\begin{array}{l}|\pi \pi\rangle_{0} \\ |\pi \pi\rangle_{1} \\ |\pi \pi\rangle_{2}\end{array}\right)=\left(\begin{array}{ccc}-\frac{1}{\sqrt{3}} & -\frac{1}{\sqrt{3}} & -\frac{1}{\sqrt{3}} \\ -\frac{1}{\sqrt{2}} & \frac{1}{\sqrt{2}} & 0 \\ -\frac{1}{\sqrt{6}} & -\frac{1}{\sqrt{6}} & \sqrt{\frac{2}{3}}\end{array}\right)\left(\begin{array}{c}\left|\pi^{+} \pi^{-}\right\rangle \\ \left|\pi^{-} \pi^{+}\right\rangle \\ \left|\pi^{0} \pi^{0}\right\rangle\end{array}\right)$

$|\pi \eta\rangle_{1}=\left|\pi^{0} \eta\right\rangle$

$|27,2,1\rangle=|K K\rangle$

$\left|27,1, \frac{3}{2}\right\rangle=\frac{1}{\sqrt{2}}(|K \pi\rangle+|\pi K\rangle)$

$\left|27,1, \frac{1}{2}\right\rangle=\frac{1}{2 \sqrt{5}}(|K \pi\rangle+|\pi K\rangle-3|K \eta\rangle-3|\eta K\rangle)$

$|27,0,2\rangle=|\pi \pi\rangle$

$|27,0,1\rangle=\frac{1}{\sqrt{5}}(|K \bar{K}\rangle+|\bar{K} K\rangle)+\frac{3}{\sqrt{30}}(|\pi \eta\rangle+|\eta \pi\rangle)$

$|27,0,0\rangle=\frac{3}{2 \sqrt{15}}(-|K \bar{K}\rangle-|\bar{K} K\rangle)+\frac{1}{2 \sqrt{10}}|\pi \pi\rangle-$

$\frac{9}{2 \sqrt{30}}|\eta \eta\rangle$

$\left|\overline{10}, 1, \frac{1}{2}\right\rangle=\frac{1}{2}(|K \pi\rangle-|\pi K\rangle+|K \eta\rangle-|\eta K\rangle)$

$|\overline{10}, 0,1\rangle=\frac{1}{\sqrt{6}}(|K \bar{K}\rangle-|\bar{K} K\rangle-|\pi \pi\rangle)+\frac{1}{2} \times$

$(|\pi \eta\rangle-|\eta \pi\rangle)$

$\left|10,1, \frac{3}{2}\right\rangle=\frac{1}{\sqrt{2}}(-|K \pi\rangle+|\pi K\rangle)$

$|10,0,1\rangle=\frac{1}{\sqrt{6}}(-|K \bar{K}\rangle+|\bar{K} K\rangle+|\pi \pi\rangle)+\frac{1}{2} \times$

$(|\pi \eta\rangle-|\eta \pi\rangle)$

$\left|8_{S}, 1, \frac{1}{2}\right\rangle=\frac{1}{2 \sqrt{5}}(-3|K \pi\rangle-3|\pi K\rangle-|K \eta\rangle-|\eta K\rangle)$

$\left|8_{S}, 0,1\right\rangle=\frac{3}{\sqrt{30}}(-|K \bar{K}\rangle-|\bar{K} K\rangle)+\frac{1}{\sqrt{5}}(|\pi \eta\rangle+|\eta \pi\rangle)$

$\left|8_{S}, 0,0\right\rangle=\frac{1}{\sqrt{10}}(-|K \bar{K}\rangle-|\bar{K} K\rangle)+\frac{3}{\sqrt{15}}|\pi \pi\rangle+\frac{1}{\sqrt{5}}|\eta \eta\rangle$

$\left|8_{A}, 1, \frac{1}{2}\right\rangle=\frac{1}{2}(-|K \pi\rangle+|\pi K\rangle+|K \eta\rangle-|\eta K\rangle)$

$\left|8_{A}, 0,1\right\rangle=\frac{1}{\sqrt{6}}(|K \bar{K}\rangle-|\bar{K} K\rangle+2|\pi \pi\rangle)$

$\left|8_{A}, 0,0\right\rangle=\frac{1}{\sqrt{2}}(-|K \bar{K}\rangle+|\bar{K} K\rangle)$

$|1,0,0\rangle=\frac{1}{2}(-|K \bar{K}\rangle-|\bar{K} K\rangle)-\frac{3}{2 \sqrt{6}}|\pi \pi\rangle+\frac{1}{2 \sqrt{2}}|\eta \eta\rangle$

\section{APPENDIX C: ISOLATING THE $J / \psi$ CONTRIBUTION FROM $\mathcal{L}_{3}$}

The $\mathcal{L}_{3}$ Lagrangian in Eq. (19) has two terms. The first one contains just hadronic currents where the initial and final state have the same electric charge and, therefore, exchange neutral vector mesons only. The other term has contributions from both charged and neutral vector mesons; from this second term first one should isolate the contribution from neutral vector mesons. Let us add and subtract the appropriate term,

$$
\begin{aligned}
\operatorname{Tr}\left(J_{3 \overline{3} \mu} J_{3 \overline{3}}^{\mu}\right) \rightarrow & \operatorname{Tr}\left(J_{3 \overline{3} \mu} J_{3 \overline{3}}^{\mu}\right)-\left(J_{D_{s} \bar{D}_{s} \mu} J_{D_{s} \bar{D}_{s}}^{\mu}+J_{D^{+} D^{-} \mu} J_{D^{+} D^{-}}^{\mu}\right. \\
& \left.+J_{D^{0} \bar{D}^{0} \mu} J_{D^{0} \bar{D}^{0}}^{\mu}\right)+\left(J_{D_{s} \bar{D}_{s} \mu} J_{D_{s} \bar{D}_{s}}^{\mu}\right. \\
& \left.+J_{D^{+} D^{-} \mu} J_{D^{+} D^{-}}^{\mu}+J_{D^{0} \bar{D}^{0} \mu} J_{D^{0} \bar{D}^{0}}^{\mu}\right), \quad \text { (C1) }
\end{aligned}
$$

such that now the sum of the first two terms in Eq. (C1) has no contribution from heavy vector meson which is now in the third term alone.

The second term of Lagrangian $\mathcal{L}_{3}$ in Eq. (19) will then be expanded in order to identify terms where equal hadronic currents are connected and terms where different ones are connected:

$$
\begin{aligned}
J_{\overline{3} 3 \mu} J_{\overline{3} 3}^{\mu}= & 2\left(J_{D_{s} \bar{D}_{s} \mu}\left(J_{D^{+} D^{-}}^{\mu}+J_{D^{0} \bar{D}^{0}}^{\mu}\right)+J_{D^{+} D^{-} \mu} J_{D^{0} \bar{D}^{0}}^{\mu}\right) \\
& +J_{D_{s} \bar{D}_{s} \mu} J_{D_{s} \bar{D}_{s}}^{\mu}+J_{D^{+} D^{-} \mu} J_{D^{+} D^{-}}^{\mu} \\
& +J_{D^{0} \bar{D}^{0} \mu} J_{D^{0} \bar{D}^{0}}^{\mu} .
\end{aligned}
$$

Now terms with the product of equal neutral hadronic currents are to be multiplied by the correction $\psi_{3}$ and terms connecting different ones by $\psi_{5}$, given in Eqs. (26) and (27). As a result,

$$
\begin{aligned}
\mathcal{L}_{3}= & \operatorname{Tr}\left(J_{3 \overline{3} \mu} J_{3 \overline{3}}^{\mu}\right)-\left(J_{D_{s} \bar{D}_{s} \mu} J_{D_{s} \bar{D}_{s}}^{\mu}+J_{D^{+} D^{-} \mu} J_{D^{+} D^{-}}^{\mu}\right. \\
& \left.+J_{D^{0} \bar{D}^{0} \mu} J_{D^{0} \bar{D}^{0}}^{\mu}\right)+2 \psi_{5}\left(J_{D_{s} \bar{D}_{s} \mu}\left(J_{D^{+} D^{-}}^{\mu}+J_{D^{0} \bar{D}^{0}}^{\mu}\right)\right. \\
& \left.+J_{D^{+} D^{-} \mu} J_{D^{0} \bar{D}^{0}}^{\mu}\right)+2 \psi_{3}\left(J_{D_{s} \bar{D}_{s} \mu} J_{D_{s} \bar{D}_{s}}^{\mu}\right. \\
& \left.+J_{D^{+} D^{-} \mu} J_{D^{+} D^{-}}^{\mu}+J_{D^{0} \bar{D}^{0} \mu} J_{D^{0} \bar{D}^{0}}^{\mu}\right)
\end{aligned}
$$

One can work it out:

$$
\begin{aligned}
\mathcal{L}_{3}= & \operatorname{Tr}\left(J_{3 \overline{3} \mu} J_{3 \overline{3}}^{\mu}\right)+2 \psi_{5}\left(J_{D_{s} \bar{D}_{s} \mu}\left(J_{D^{+} D^{-}}^{\mu}+J_{D^{0} \bar{D}^{0}}^{\mu}\right)\right. \\
& \left.+J_{D^{+} D^{-} \mu} J_{D^{0} \bar{D}^{0}}^{\mu}\right)+\underbrace{\left(2 \psi_{3}-1\right)}_{\psi_{5}}\left(J_{D_{s} \bar{D}_{s} \mu} J_{D_{s} \bar{D}_{s}}^{\mu}\right. \\
& \left.+J_{D^{+} D^{-} \mu} J_{D^{+} D^{-}}^{\mu}+J_{D^{0} \bar{D}^{0} \mu} J_{D^{0} \bar{D}^{0}}^{\mu}\right) \\
= & \operatorname{Tr}\left(J_{3 \overline{3} \mu} J_{3 \overline{3}}^{\mu}\right)+\psi_{5}\left(J_{D_{s} \bar{D}_{s} \mu} J_{D_{s} \bar{D}_{s}}^{\mu}+J_{D^{+} D^{-} \mu} J_{D^{+} D^{-}}^{\mu}\right. \\
& +J_{D^{0} \bar{D}^{0} \mu} J_{D^{0} \bar{D}^{0}}^{\mu}+2 J_{D_{s} \bar{D}_{s} \mu}\left(J_{D^{+} D^{-}}^{\mu}+J_{D^{0} \bar{D}^{0}}^{\mu}\right) \\
& \left.+2 J_{D^{+} D^{-} \mu} J_{D^{0} \bar{D}^{0}}^{\mu}\right)=\operatorname{Tr}\left(J_{3 \overline{3} \mu} J_{3 \overline{3}}^{\mu}\right)+\psi_{5} J_{\overline{3} 3 \mu} J_{\overline{3} 3}^{\mu} .
\end{aligned}
$$

And this is the simple form we write down in Eq. (28). Yet, in the amplitudes we use the $\psi_{3}$ and $\psi_{5}$ factors. 
[1] B. Aubert et al. (BABAR Collaboration), Phys. Rev. Lett. 90, 242001 (2003).

[2] P. Krokovny et al. (Belle Collaboration), Phys. Rev. Lett. 91, 262002 (2003).

[3] K. Abe et al. (Belle Collaboration), Phys. Rev. D 69, 112002 (2004).

[4] S. Godfrey and N. Isgur, Phys. Rev. D 32, 189 (1985).

[5] G. S. Bali, Phys. Rev. D 68, 071501 (2003).

[6] K. Terasaki, Phys. Rev. D 68, 011501 (2003).

[7] Z. G. Wang and S. L. Wan, Nucl. Phys. A778, 22 (2006).

[8] J. Vijande, F. Fernandez, and A. Valcarce, Phys. Rev. D 73, 034002 (2006); 74, 059903(E) (2006).

[9] A. P. Szczepaniak, Phys. Lett. B 567, 23 (2003).

[10] T. Barnes, F. E. Close, and H. J. Lipkin, Phys. Rev. D 68, 054006 (2003).

[11] F. P. Sassen and S. Krewald, Int. J. Mod. Phys. A 20, 705 (2005).

[12] E. S. Swanson, Phys. Rep. 429, 243 (2006).

[13] A. A. Petrov, in Proceedings of Flavor Physics and CP Violation (FPCP 2003), Paris, France, 2003, pp MEC05 [arXiv:hep-ph/0311371].

[14] P. Colangelo, F. De Fazio, and R. Ferrandes, Mod. Phys. Lett. A 19, 2083 (2004).

[15] E. E. Kolomeitsev and M. F. M. Lutz, Phys. Lett. B 582, 39 (2004).

[16] J. Hofmann and M.F. M. Lutz, Nucl. Phys. A733, 142 (2004).

[17] F. K. Guo, P. N. Shen, H. C. Chiang, and R. G. Ping, Phys. Lett. B 641, 278 (2006).

[18] N. Isgur and M. B. Wise, Phys. Lett. B 232, 113 (1989).

[19] N. Isgur and M. B. Wise, Phys. Lett. B 237, 527 (1990).

[20] N. Isgur and M. B. Wise, Phys. Rev. Lett. 66, 1130 (1991).

[21] M. B. Wise, Phys. Rev. D 45, R2188 (1992).

[22] J. A. Oller and E. Oset, Nucl. Phys. A620, 438 (1997); A652, 407 (1999).

[23] J. A. Oller, E. Oset, and J. R. Pelaez, Phys. Rev. D 59, 074001 (1999); 60, 099906(E) (1999).

[24] A. Gomez Nicola and J. R. Pelaez, Phys. Rev. D 65, 054009 (2002).
[25] N. Kaiser, Eur. Phys. J. A 3, 307 (1998).

[26] J. Nieves and E. Ruiz Arriola, Phys. Lett. B 455, 30 (1999).

[27] V. E. Markushin, Eur. Phys. J. A 8, 389 (2000).

[28] J. R. Pelaez, AIP Conf. Proc. 892, 72 (2007).

[29] H. Leutwyler, arXiv:hep-ph/0612112.

[30] J. Gasser and H. Leutwyler, Nucl. Phys. B250, 465 (1985).

[31] J. A. Oller, E. Oset, and A. Ramos, Prog. Part. Nucl. Phys. 45, 157 (2000).

[32] G. Pari, B. Schwesinger, and H. Walliser, Phys. Lett. B 255, 1 (1991).

[33] S. T. Hong and Y. J. Park, Phys. Rev. D 63, 054018 (2001).

[34] H. Walliser, Nucl. Phys. A548, 649 (1992).

[35] W. M. Yao et al. (Particle Data Group), J. Phys. G 33, 1 (2006).

[36] A. X. El-Khadra, A. S. Kronfeld, P. B. Mackenzie, S. M. Ryan, and J. N. Simone, Phys. Rev. D 58, 014506 (1998).

[37] J. Hofmann and M.F.M. Lutz, Nucl. Phys. A763, 90 (2005).

[38] J. E. Palomar and E. Oset, Nucl. Phys. A716, 169 (2003).

[39] E. Oset and A. Ramos, Nucl. Phys. A635, 99 (1998).

[40] J. A. Oller and E. Oset, Phys. Rev. D 60, 074023 (1999).

[41] T. Inoue, E. Oset, and M. J. Vicente Vacas, Phys. Rev. C 65, 035204 (2002).

[42] E. van Beveren, D. V. Bugg, F. Kleefeld, and G. Rupp, Phys. Lett. B 641, 265 (2006).

[43] D. V. Bugg, Eur. Phys. J. C 47, 57 (2006).

[44] D. V. Bugg, Eur. Phys. J. C 47, 57 (2006).

[45] D. V. Bugg, Eur. Phys. J. A 25, 107 (2005); 26, 151 (2005).

[46] F. K. Guo, R. G. Ping, P. N. Shen, H. C. Chiang, and B. S. Zou, Nucl. Phys. A773, 78 (2006).

[47] I. Bediaga (Fermilab E791 Collaboration), arXiv:hep-ex/ 0208039.

[48] Y. J. Zhang, H. C. Chiang, P. N. Shen, and B. S. Zou, Phys. Rev. D 74, 014013 (2006).

[49] J. J. de Swart, Rev. Mod. Phys. 35, 916 (1963).

[50] T. A. Kaeding, arXiv:nucl-th/9502037. 